\title{
Critical window for the vacant set left by random walk on the configuration model
}

\author{
Jiří Černý, Thomas Hayder
}

Departement Mathematik und Informatik

Preprint No. 2021-16

Fachbereich Mathematik

July 2021

Universität Basel

CH-4051 Basel

dmi.unibas.ch 


\title{
Critical window for the vacant set left by random walk on the configuration model
}

\author{
Jiří Černý and Thomas Hayder \\ Department of Mathematics and Computer Science, University of Basel
}

July 21, 2021

\begin{abstract}
We study the simple random walk on the configuration model with given degree sequence $\left(d_{1}^{n}, \ldots, d_{n}^{n}\right)$ and investigate the connected components of its vacant set at level $u>0$. We show that the size of the maximal connected component exhibits a phase transition at level $u^{*}$ which can be related with the critical parameter of random interlacements on a certain Galton-Watson tree. We further show that there is a critical window of size $n^{-1 / 3}$ around $u^{*}$ in which the largest connected components of the vacant set have a metric space scaling limit resembling the one of the critical Erdôs-Rényi random graph.
\end{abstract}

\section{Introduction}

We consider the vacant set of the random walk on the configuration model and study its percolative properties. The main goal is to understand the maximal connected components of this set in the vicinity of the critical threshold. We will show that the model belongs to the Erdôs-Rényi universality class, that is its behaviour is similar to the classical critical Erdôs-Rényi graph. In particular we show the conjecture of [BS20] about the scaling limit for the metric space structure of the maximal connected components of the vacant set in the critical regime.

We start with a precise definition of the model. For every $n \in \mathbb{N}$, let $\mathbf{d}^{n}=\left(d_{1}^{n}, \ldots, d_{n}^{n}\right)$ be a degree sequence such that the total degree $L_{n}=\sum_{x \in[n]} d_{x}^{n}$ is even. We use $\mathcal{G}^{\mathbf{d}^{n}}$ to denote the set of all multigraphs with the vertex set $[n]=\{1,2, \ldots, n\}$ such that the degree of every $x \in[n]$ is $d_{x}^{n}$ (loops are counted twice), and write $\mathbb{P}^{\mathbf{d}^{n}}$ for the law on $\mathcal{G}^{\mathbf{d}^{n}}$ of the configuration model with the degree sequence $\mathbf{d}^{n}$, that is of the random multigraph $G_{n}=\left([n], E_{n}\right)$ obtained by the usual pairing construction (see Section 2.2 for the details). For an arbitrary finite multigraph $G$, let $\lambda_{G}$ denote its spectral gap (see (2.7) for the definition). In addition, let $n_{i}\left(\mathbf{d}^{n}\right)$ be the number of vertices $x \in[n]$ with $d_{x}^{n}=i, i \in \mathbb{N}$. Through the paper we assume that the degree sequence $\mathbf{d}^{n}$ has the following properties:

Assumption 1.1. There exist finite positive constants $\Delta \geq 3, c, C, \varepsilon_{1}, \varepsilon_{2}$ and $l \geq 0$, and a probability mass function $p=\left(p_{i}\right)_{1 \leq i \leq \Delta}$ on $\{1, \ldots, \Delta\}$ with $p_{1}=p_{2}=0$ such that the following three conditions are satisfied:

(a) $1 \leq d_{x}^{n} \leq \Delta$ for all $x \in[n]$,

(b) $\left|n_{i}\left(\mathbf{d}^{n}\right)-n p_{i}\right| \leq C n^{\frac{1}{3}-\varepsilon_{1}}$ for all $i \in\{1,2, \ldots, \Delta\}$, 
(c) $\mathbb{P}^{\mathbf{d}^{n}}\left[\lambda_{G_{n}} \geq c \log ^{-l} n\right] \geq 1-C n^{-\frac{2}{3}-\varepsilon_{2}}$.

Given a fixed realisation of $G_{n} \in \mathcal{G}^{\mathbf{d}^{n}}$, let $P^{G_{n}}$ be the distribution on $[n]^{\mathbb{N}}$ of the canonical lazy discrete-time simple random walk $X=\left(X_{k}\right)_{k \geq 0}$ on $G_{n}$, which is started from its stationary distribution $\pi^{G_{n}}$, where $\pi^{G_{n}}(x)=d_{x}^{n} / L_{n}$ for $x \in[n]$. We endow $[n]^{\mathbb{N}}$ with the product $\sigma$-algebra $\mathcal{F}_{n}$. We further write $\mathbf{P}_{n}$ for the joint distribution of $\left(G_{n}, X\right)$ on $\mathcal{G}^{\mathbf{d}^{n}} \times[n]^{\mathbb{N}}$ given by

$$
\mathbf{P}_{n}\left[G_{n} \in A, X \in B\right]=\int_{G_{n} \in A} P^{G_{n}}[X \in B] \mathbb{P}^{\mathbf{d}^{n}}\left[d G_{n}\right] \quad A \subset \mathcal{G}^{\mathbf{d}^{n}}, B \in \mathcal{F}_{n} .
$$

The vacant set of the random walk on $G_{n}$ at the level $u \geq 0$ is the set of vertices not visited by the simple random walk up to time $2 u m_{p} n$,

$$
\mathcal{V}_{G_{n}}^{u}=[n] \backslash\left\{X_{k}: 0 \leq k \leq 2 u m_{p} n\right\},
$$

where $m_{p}=\sum_{i=3}^{\Delta} i p_{i}$ is the expectation of $p$. The scaling factor $2 m_{p}$ is chosen for convenience to match the critical points of various models appearing below, we refer to Remark 1.7 for more details.

Our goal is to investigate the connectivity properties of the subgraph of $G_{n}$ induced by $\mathcal{V}_{G_{n}}^{n}$. To this end, let $\mathcal{C}_{j}^{u}(n)$ denote the $j$-th largest connected component of this subgraph and let $\left|\mathcal{C}_{j}^{u}(n)\right|$ be the number of its vertices. Our first main result is the following theorem implying the phase transition in the behaviour of $\left|\mathcal{C}_{1}^{u}(n)\right|$.

Theorem 1.2. For every degree sequence $\mathbf{d}^{n}$ satisfying Assumption 1.1, there is a constant $u^{*} \in(0, \infty)$, such that the following holds:

(a) (Supercritical regime) For $u<u^{*}$, there exists a constant $\rho=\rho(u, p) \in(0,1)$, characterised in Remark 5.2 below, such that for every $\varepsilon>0$

$$
\lim _{n \rightarrow \infty} \mathbf{P}_{n}\left[\left|\frac{\left|\mathcal{C}_{1}^{u}(n)\right|}{n}-\rho\right| \leq \varepsilon\right]=1 .
$$

(b) (Subcritical regime) For $u>u^{*}$, there exists a constant $A=A(u, \Delta)$, such that

$$
\lim _{n \rightarrow \infty} \mathbf{P}_{n}\left[\left|\mathcal{C}_{1}^{u}(n)\right| \leq A \log n\right]=1 .
$$

(c) (Critical regime) Let $\left(u_{n}\right)_{n \geq 0}$ be a sequence such that for some $\eta<\infty$

$$
\left|n^{1 / 3}\left(u_{n}-u^{*}\right)\right| \leq \eta
$$

Then for every $\varepsilon>0$ there exists $A=A(\eta, \varepsilon, p)$ such that for all $n$ large enough

$$
\mathbf{P}_{n}\left[A^{-1} n^{\frac{2}{3}} \leq\left|\mathcal{C}_{1}^{u_{n}}(n)\right| \leq A n^{\frac{2}{3}}\right] \geq 1-\varepsilon .
$$

(d) The constant $u^{*}$ agrees with the critical parameter of random interlacements on the Galton-Watson tree $\mathcal{T}^{\prime}$ with offspring distribution $p^{*}$ given by

$$
p_{i}^{*}=(i+1) p_{i+1} m_{p}^{-1}, \quad i \in\{0,1, \ldots, \Delta-1\} .
$$

(See Section 2.4 for the definition of random interlacements)

Our second main result confirms the conjecture of Bhamidi and Sen (see [BS20, Conjecture 2.7]) about the convergence of the connected components $\left(\mathcal{C}_{j}^{u_{n}}(n)\right)_{j \geq 1}$, viewed as metric-measure spaces, in the critical window. 
Theorem 1.3 (Scaling limit). Let Assumption 1.1 hold and let $\left(u_{n}\right)_{n \geq 0}$ be a sequence such that there is $\eta \in \mathbb{R}$ satisfying

$$
u_{n}=u^{*}+\eta n^{-1 / 3}+o\left(n^{-1 / 3}\right) \quad \text { as } n \rightarrow \infty .
$$

Endow every component $\mathcal{C}_{j}^{u_{n}}(n)$ with its graph distance and the uniform probability measure on its vertices. Then there exists a sequence $\mathbf{M}^{p}(\eta)=\left(M_{1}^{p}(\eta), M_{2}^{p}(\eta), \ldots\right)$ of (random) compact metric-measure spaces such that under the measure $\mathbf{P}_{n}$

$$
\frac{1}{n^{1 / 3}}\left(\mathcal{C}_{1}^{u_{n}}(n), \mathcal{C}_{2}^{u_{n}}(n), \ldots\right) \stackrel{n \rightarrow \infty}{\longrightarrow} \mathbf{M}^{p}(\eta)
$$

with respect to the product topology induced by the Gromov-Hausdorff-Prokhorov distance on each coordinate. For a complete description of the limiting spaces we refer to [BS20, Construction 5.6].

Statements similar to Theorems 1.2, 1.3 are known to hold for several different sequences of quickly mixing graphs with locally tree-like structure. First, the phase transition for the vacant set of the random walk was proved for (possibly deterministic) $d$-regular large girth expanders by Černý, Teixeira and Windisch [ČTW11]. Later, in [CF13], Cooper and Frieze made the key observation that randomness of the graph greatly simplifies studying of the behaviour of the vacant set, and proved the phase transition for the vacant set of the random walk on connected Erdôs-Rényi graphs and on $d$-regular random graphs. Their technique was later extended to every supercritical Erdôs-Rényi graph by Wassmer [Was15]. Moreover, [ČTW11] and [Was15] show that the critical parameter for the phase transition coincides with the critical parameter of random interlacements on the corresponding infinite volume limit, that the $d$-regular tree or a particular Galton-Watson tree, respectively.

The behaviour of the vacant set in the vicinity of the critical point $u^{*}$ has been previously studied for the $d$-regular random graphs only. In this case, Černý and Teixeira [ČT13] showed the existence of a critical window of width $n^{-1 / 3}$ around $u^{*}$ where the size of the largest connected component is of order $n^{2 / 3}$. Later, Bhamidi and Sen [BS20] described precisely the scaling limit for all connected components, by combining their results on the behaviour of critical random graphs with a given degree sequence with the controls of the degree sequence of the vacant set obtained in [ČT13]. The scaling limit resembles the scaling limit of the components of the critical Erdôs-Rényi graph given in [ABG12].

The results of Theorems 1.2 and 1.3 naturally extend [ČT13] and [BS20]. In particular Theorem 1.3 proves and makes more precise Conjecture 2.7 of [BS20].

The proofs of the two main theorems broadly follows the strategy of [ČT13] and [BS20]: We first extend the observation of Cooper and Frieze [CF13] to our case (see Proposition 2.1) which allows us to view the vacant set of the random walk as a random graph with a random degree sequence $\mathbf{d}^{n, u}$. As a consequence, we can apply the extensive theory about such random graphs, summarized in Theorem 2.2. The main body of the paper (Sections 3 and 4) then deals with providing sufficiently strong estimates on the distribution of the sequence $\mathbf{d}^{n, u}$. Here we mainly follow the techniques from [ČT13]: We first investigate the local behaviour of the random walk, concentrating on how it visits the neighbours of a given vertex (Proposition 3.1). Then, we construct a suitable coupling of the random walk on $G_{n}$ with a random walk on the unimodular GaltonWatson tree (see Lemma 4.1), to estimate the expected degree distribution of the vacant set $\mathcal{V}_{G_{n}}^{u}$ (Theorem 4.4). Finally, we use the mixing properties of the random walk on $G_{n}$ to prove a corresponding concentration result for the degree distribution (Theorem 4.6). The main theorems are then proved in Section 5. The challenge in all these steps is to obtain sharp enough estimates that are useful also in the critical regime. 
Compared to the $d$-regular random graph case, considered in [ČT13], we have to deal with two additional difficulties. First, although the configuration model looks locally like a tree, this tree is a Galton-Watson tree, and not a $d$-regular tree like in [ČT13], which considerably complicates the necessary random walk estimates in Section 3. Second, since we allow the vertices of the graph to have degree 1 or 2 , our graphs are not as robust as the $d$-regular random graph, $d \geq 3$, under deletion of small number of vertices, which requires some additional arguments (e.g., in Lemmas 3.2, 3.3).

We close this introduction with remarks about the assumptions of our main results, their possible generalisations and the appearance of the scaling factor in definition (1.2).

Remark 1.4. (a) Assumption 1.1(b) and $p_{1}=p_{2}=0$ imply that $G_{n}$ satisfies the assumptions of [FvdH17, Theorem 2.2], and is thus connected $\mathbb{P}^{\mathbf{d}^{n}}$-a.a.s. With more technical effort it would, in principle, be possible to treat the regime when $G_{n}$ is disconnected but has a giant component $\mathbb{P}^{\mathbf{d}^{n}}$-a.a.s., as was done for the vacant set of the random walk on the largest connected component on the Erdôs-Rényi graph in [Was15].

(b) If the degree sequence is constant, that is $d_{x}^{n}=d \geq 3$, for all $x \in[n]$, Assumption 1.1 is always satisfied. Here (c) follows from [Fri91, Theorem 1.1] with $l=0$. Note that (c) is not very restrictive. Moreover, results about the mixing time of random walks on random graphs suggest, that (c) could be proved from assumptions (a) and (b) (see [BLPS18]). We do not investigate this issue in detail here.

Remark 1.5. The assumptions of Theorem 1.3 are stronger than the assumptions that appear in Conjecture 2.7 of [BS20]. Besides assuming that the maximal degree of $G_{n}$ is bounded (cf. Assumption 1.1(a)), which is purely for technical reasons, we need some control of the speed of convergence of the degree distribution to the probability mass function $p$, as in Assumption 1.1(b). Conjecture 2.7 of [BS20] only requires $\lim _{n \rightarrow \infty} n_{i}\left(\mathbf{d}^{n}\right) / n=p_{i}$ for all $i$. We believe that the conjecture does not hold under this weaker assumption since a slow convergence of $n_{i}\left(\mathbf{d}^{n}\right) / n$ to $p_{i}$ can shift the position of the critical window.

Remark 1.6. Instead of the law $\mathbb{P}^{\mathbf{d}^{n}}$ (on the set of multigraphs $\mathcal{G}^{\mathbf{d}^{n}}$ ) of the configuration model, it would be possible to consider the uniform measure $\overline{\mathbb{P}}^{d^{n}}$ on the set of all simple graphs $\overline{\mathcal{G}}^{\mathbf{d}^{n}} \subset \mathcal{G}^{\mathbf{d}^{n}}$. Under Assumption 1.1, all statements of Theorem 1.2 continue to hold for this model as well, since a $\mathbb{P}^{\mathbf{d}^{n}}$ distributed multigraph $G_{n}$ conditioned on being simple has distribution $\overline{\mathbb{P}}^{n}$ (see e.g. [vdH17a, Proposition 7.15]),

$$
\mathbb{P}^{\mathbf{d}^{n}}\left[G_{n} \in \cdot \mid G_{n} \in \overline{\mathcal{G}}^{\mathbf{d}^{n}}\right]=\overline{\mathbb{P}}^{\mathbf{d}^{n}}\left[G_{n} \in \cdot\right]
$$

and the probability of a multigraph $G_{n}$ being simple has a positive limit (see e.g. [vdH17a, Theorem 7.12]),

$$
\lim _{n \rightarrow \infty} \mathbb{P}^{\mathbf{d}^{n}}\left[G_{n} \in \overline{\mathcal{G}}^{\mathbf{d}^{n}}\right]=c>0 .
$$

Extending Theorem 1.3 to this case is not so straightforward and would require additional arguments.

Remark 1.7. Recall the scaling factor $2 m_{p}$ in the definition (1.2) of the vacant set. The purpose of this choice is to force the critical values $u^{*}$ of Theorem 1.2 and the critical value $u^{*}$ of random interlacements on the unimodular Galton-Watson tree in Proposition 2.3 below to be equal. In fact, the definition of random interlacements on this tree contains a free scaling parameter, which can be interpreted as a weight of the edges of the tree. In (2.27) we endow every edge with weight one (similarly as in [Tas10]). We could alternatively define the capacity as

$$
\operatorname{cap}_{\mathcal{T}}(K)=\sum_{x \in K}\left(2 m_{p}\right)^{-1} d_{x} P_{x}^{\mathcal{T}}\left[\widetilde{H}_{K}=\infty\right],
$$

corresponding to a weighting by $\left(2 m_{p}\right)^{-1}$, which would eliminate the scaling factor $2 m_{p}$ in (1.2). 


\section{Definitions and preliminaries}

In this section we introduce the notation used through the paper and recall some known results. We use $c, c^{\prime}$, etc. to denote finite positive constants whose value might change during the computations. For every set $A$, we write $|A|$ for its cardinality, and use the standard $o(\cdot)$ and $O(\cdot)$ notation. For a sequence of probability measures $P_{n}$ and events $A_{n}$, we say $A_{n}$ holds $P_{n}$-a.a.s, if $\lim _{n \rightarrow \infty} P_{n}\left[A_{n}\right]=1$.

\subsection{Graphs and random walk}

Let $G=(V, E)$ be a multigraph. We always endow it with the usual graph distance $\operatorname{dist}_{G}(\cdot, \cdot)$, and denote $\operatorname{diam}(G)$ its diameter. The ball in $G$ centred at $x \in V$ with radius $r$ is denoted by $B(x, r)$. If $(x, y)$ is an edge of $G$, then we write $x \sim y . d_{x}$ stands for the degree of $x \in V$ in $G$, that is $d_{x}=\sum_{y \in V} n_{x y}$, where $n_{x y}$ is the number of edges connecting $x$ and $y$ in $G$ (the loops are counted twice in $n_{x x}$ ). The set

$$
\partial_{e} A=\left\{(x, y) \in E: x \in A, y \in A^{c}\right\}
$$

is called the edge boundary of the set $A$. When no confusion can arise, we identify the subset $A \subset V$ with the corresponding induced subgraph of $G$.

For an arbitrary finite multigraph $G=(V, E)$, we use $P_{x}^{G}$ to denote the distribution on $V^{\mathbb{N}}$ of the canonical lazy discrete-time simple random walk $X=\left(X_{k}\right)_{k \geq 0}$ on $G$ started at $x \in V$, that is of the Markov chain with generator given by

$$
\mathcal{L} f(x)=\sum_{y \in V} p_{x y}(f(y)-f(x)), \quad \text { for } f: V \rightarrow \mathbb{R}, \quad x \in V,
$$

where

$$
p_{x y}= \begin{cases}\frac{n_{x y}}{2 d_{x}}, & x \neq y, \\ \frac{1}{2}+\frac{n_{x y}}{2 d_{x}}, & x=y .\end{cases}
$$

We write $E_{x}^{G}$ for the corresponding expectation. For a measure $\mu$ on $V$, we define

$$
P_{\mu}^{G}[\cdot]=\sum_{x \in G} \mu(x) P_{x}^{G}[\cdot]
$$

For a set $A \subset V$, we denote by $\widetilde{H}_{A}$ and $H_{A}$ the respective hitting and entrance time of $A$,

$$
\widetilde{H}_{A}=\inf \left\{k \geq 1: X_{k} \in A\right\} \quad \text { and } \quad H_{A}=\inf \left\{k \geq 0: X_{k} \in A\right\} .
$$

For all real valued functions $f, g$ on $V$, we define the Dirichlet form

$$
\mathcal{D}(f, g)=\frac{1}{2} \sum_{x, y \in V} \pi^{G}(x) p_{x y}(f(x)-f(y))(g(x)-g(y))
$$

where $\pi^{G}(x)=d_{x} / \sum_{y \in V} d_{y}$ is the stationary distribution of the random walk $X$. The spectral gap of $X$ is given by

$$
\lambda_{G}=\min \left\{\mathcal{D}(f, f): \pi^{G}\left(f^{2}\right)=1, \pi^{G}(f)=0\right\} .
$$

Finally, we define the edge expansion $h(G)$ of $G$ by

$$
h(G)=\min \left\{\frac{Q(A)}{\pi^{G}(A)}: A \subset V, \pi^{G}(A) \leq \frac{1}{2}\right\}, \quad \text { where } \quad Q(A)=\sum_{(x, y) \in \partial_{e} A} \pi^{G}(x) p_{x y} .
$$


The Cheeger inequality (see [SC97, Lemma 3.3.7]) relates the spectral gap $\lambda_{G}$ to the edge expansion $h(G)$. For some $c, c^{\prime} \in(0, \infty)$,

$$
\operatorname{ch}(G)^{2} \leq \lambda_{G}<c^{\prime} h(G)
$$

We frequently use the following standard convergence result for reversible Markov chains (see e.g. [LPW09, Theorem 12.3]). For all $k \geq 0$,

$$
\sup _{x, y \in V}\left|P_{x}^{G}\left[X_{k}=y\right]-\pi^{G}(y)\right| \leq\left(\min _{x \in V} \pi^{G}(x)\right)^{-1} \exp \left(-\lambda_{G} k\right) .
$$

\subsection{Configuration model and vacant graph}

In [CF13], Cooper and Frieze make the observation that, for the $d$-regular random graph, the graph induced by the vacant set of random walk is distributed as a random graph with a (random) degree sequence. This observation extends straightforwardly to our setting. For readers convenience we provide its proof in Proposition 2.1 below.

To state this proposition it is suitable to introduce the vacant graph $\mathbf{V}^{n, u}$. For $G_{n}=\left([n], E_{n}\right)$, let

$$
\mathbf{V}^{n, u}=\left([n], E^{u}\right), \quad \text { where } \quad E^{u}=\left\{(x, y) \in E_{n}: x, y \in \mathcal{V}_{G_{n}}^{u}\right\},
$$

and let $\mathcal{Q}_{n}^{u}$ be the distribution on $\{0, \ldots, \Delta\}^{n}$ of its degree sequence

$$
\mathbf{d}^{n, u}=\left(d_{1}^{n, u}, d_{2}^{n, u}, \ldots, d_{n}^{n, u}\right)
$$

under the annealed measure $\mathbf{P}_{n}$. Note that the non-trivial connected components of the vacant graph $\mathbf{V}^{n, u}$ (that is those having at least two vertices) coincide with the connected components of the subgraph of $G_{n}$ induced by the vacant set $\mathcal{V}_{G_{n}}^{u}$.

Proposition 2.1. For every $u \geq 0$, the distribution of the vacant graph $\mathbf{V}^{n, u}$ under $\mathbf{P}_{n}$ satisfies

$$
\mathbf{P}_{n}\left[\mathbf{V}^{n, u} \in \cdot\right]=\int \mathbb{P}^{\mathbf{d}^{n, u}}\left[G_{n} \in \cdot\right] \mathcal{Q}_{n}^{u}\left(d \mathbf{d}^{n, u}\right),
$$

where $\mathbb{P}^{\mathbf{d}^{n, u}}$ is the distribution of the multigraph with the degree sequence $\mathbf{d}^{n, u}$.

Before proving Proposition 2.1, let us recall in more detail the standard pairing construction of the multigraph $G_{n}$ with the degree sequence $\mathbf{d}^{n}$ : We associate every vertex $x \in[n]$ with $d_{x}^{n}$ half-edges and denote by

$$
H^{n}=\left\{\left(x, i \mid: x \in[n], i \in\left[d_{x}^{n}\right]\right\}\right.
$$

the set of all these half-edges. Note that $\left|H^{n}\right|=L_{n}=\sum_{x \in[n]} d_{x}^{n}$. The $\mathbb{P}^{\mathbf{d}^{n}}$-distributed multigraph $G_{n}$ is obtained by sampling a random perfect matching $M$ of $H^{n}$ (that is, a partitioning of $H^{n}$ into $L_{n} / 2$ disjoint pairs) uniformly from the set of all such matchings, and by setting $G_{n}=G_{n}^{M}:=\left([n], E_{M}\right) \in \mathcal{G}^{\mathbf{d}^{n}}$ with

$$
E_{M}=\left\{(x, y):\left\{\left(x, i \mid,(y, j \mid\} \in M \text { for some } i \in\left[d_{x}^{n}\right], j \in\left[d_{y}^{n}\right]\right\} .\right.\right.
$$

It is well known that a uniform random matching $M$ and so the corresponding multigraph $G_{n}^{M}$ can be constructed sequentially. We start with the empty matching $\bar{M}=\varnothing$ and repeat the following steps until we obtain a full matching: Given a partial matching $\bar{M} \subset M$ and the corresponding set of half-edges $H^{\bar{M}}=\{(x, i \mid:(x, i \mid$ is matched in $\bar{M}\}$, we choose two half-edges $\left(x, i \mid,\left(y, j \mid \in H^{n} \backslash H^{\bar{M}}\right.\right.$; the first half-edge $(x, i \mid$ can be chosen by an arbitrary rule $R$, the second half-edge $(y, j \mid$ is chosen uniformly from the set of all 
remaining half-edges $H^{n} \backslash\left(H^{\bar{M}} \cup(x, i \mid)\right.$. As last step we match the half-edges $(x, i \mid$ and $(y, j \mid$ and add $\{(x, i \mid,(y, j \mid\}$ to the partial matching $\bar{M}$.

It follows that, conditioned on the partial matching $\bar{M}, M \backslash \bar{M}$ is distributed as a uniform perfect matching of $H^{n} \backslash H^{\bar{M}}$. Therefore, the corresponding multigraphs satisfy

$$
\mathbb{P}^{\mathbf{d}^{n}}\left[G_{n}^{M \backslash \bar{M}} \in \cdot \mid \bar{M} \subset M\right]=\mathbb{P}^{\mathbf{d}^{n}(\bar{M})}[G \in \cdot],
$$

where $d_{x}^{n}(\bar{M})$ is the number of half-edges incident to $x$ in $H^{n}$ that are not yet matched in $\bar{M}$, and $G_{n}^{M \backslash \bar{M}}$ is the graph corresponding to a non-perfect matching $M \backslash \bar{M}$.

This sequential construction will also be applied later to construct a local coupling of the multigraph $G_{n}$ with an infinite tree. In this case the random walk $X$ will be used to define the rule $R$.

Proof of Proposition 2.1. The proof follows the steps of [ČT13, Proposition 2.1]. Let $M$ be a uniformly distributed matching of $H^{n}$ and let $X$ be a random walk on $G_{n}=G_{n}^{M}$. Define $M^{u} \subset M$ to be the set of all pairs of half-edges incident to a vertex visited by $X$ up to time $2 u m_{p} n$ (cf. (1.2)), that is

$$
M^{u}=\left\{\left\{\left(x, i \mid,(y, j \mid\} \in M: x \in\left\{X_{k}: k \leq 2 u m_{p} n\right\}, i \in\left[d_{x}^{n}\right]\right\} .\right.\right.
$$

The vacant graph $\mathbf{V}^{n, u}$ then agrees with $G^{M \backslash M^{u}}$. In particular, $d_{x}^{n, u}$ (see (2.12)) is the number of the half-edges incident to $x$ not matched in $M^{u}$. Denoting by $\mathcal{F}^{u}$ the $\sigma$-algebra generated by $\left\{M^{u}, X_{k}, k \leq 2 u m_{p} n\right\}$, the above implies that $\mathbf{d}^{n, u}$ is $\mathcal{F}^{u}$-measurable. From (2.16) it follows that, conditionally on $\mathcal{F}^{u}$, the distribution of $G^{M \backslash M^{u}}$ only depends on the half-edges not matched in $M^{u}$, and is given by $\mathbb{P}^{\mathbf{d}^{n, u}}$. More precisely,

$$
\mathbf{P}_{n}\left[\mathbf{V}^{n, u} \in \cdot \mid \mathcal{F}^{u}\right]=\mathbf{P}_{n}\left[G^{M \backslash M^{u}} \in \cdot \mid \mathbf{d}^{n, u}\right]=\mathbb{P}^{\mathbf{d}^{n, u}}[G \in \cdot] .
$$

Integrating this equality and using the definition of $\mathcal{Q}_{n}^{u}$ then implies

$$
\begin{aligned}
\mathbf{P}_{n}\left[\mathbf{V}^{n, u} \in \cdot\right] & =\mathbf{P}_{n}\left[\mathbf{P}_{n}\left[\mathbf{V}^{n, u} \in \cdot \mid \mathcal{F}^{u}\right]\right]=\mathbf{P}_{n}\left[\mathbb{P}^{\mathbf{d}^{n, u}}[G \in \cdot]\right] \\
& =\int \mathbb{P}^{\mathbf{d}^{n, u}}\left[G_{n} \in \cdot\right] \mathcal{Q}_{n}^{u}\left(d \mathbf{d}^{n, u}\right),
\end{aligned}
$$

which completes the proof.

\subsection{Configuration model and its connected components}

In this section we summarize known results from the theory of the configuration model with the given degree sequence $\mathbf{d}^{n}=\left(d_{1}^{n}, \ldots, d_{n}^{n}\right)$. It turns out that their connectivity behaviour essentially depends only on one parameter

$$
Q\left(\mathbf{d}^{n}\right)=\frac{\sum_{\left(x, i \mid \in H^{n}\right.} d_{x}^{n}}{L_{n}}=\frac{\sum_{x \in[n]}\left(d_{x}^{n}\right)^{2}}{\sum_{x \in[n]} d_{x}^{n}} .
$$

In the following theorem we use $\mathcal{C}_{j}\left(G_{n}\right)$ to denote the $j$-th largest connected component of the multigraph $G_{n}$. We also recall that $n_{i}\left(\mathbf{d}^{n}\right)=\left|\left\{x \in[n]: d_{x}^{n}=i\right\}\right|$, as in Section 1 .

Theorem 2.2. Let $\left(\mathbf{d}^{n}\right)_{n \geq 1}$ be a sequence of degree sequences, such that for all $n \in \mathbb{N}$ $\max _{1 \leq x \leq n}\left\{d_{x}^{n}\right\} \leq \Delta<\infty, n_{1}\left(\mathbf{d}^{n}\right) \geq \zeta$ for some $\zeta>0$, and for some probability mass function $p=\left(p_{i}\right)_{0 \leq i \leq \Delta}$

$$
\lim _{n \rightarrow \infty} \frac{n_{i}\left(\mathbf{d}^{n}\right)}{n}=p_{i}, \quad \text { for all } 0 \leq i \leq \Delta .
$$


(a) (Supercritical regime) Let $\lim _{n \rightarrow \infty} Q\left(\mathbf{d}^{n}\right)=Q_{\infty}>2$. Denote $g(x)=\sum_{i=0}^{\Delta} p_{i} x^{i}$ the generating function of $p$. Then,

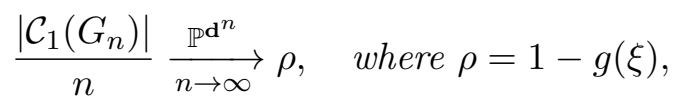

and $\xi$ is the unique solution in $(0,1)$ to $g^{\prime}(\xi)=\xi \sum_{i=0}^{\Delta} i p_{i}$.

(b) (Subcritical regime) Let $\lim _{n \rightarrow \infty} Q\left(\mathbf{d}^{n}\right)=Q_{\infty}<2$. Then there exists a constant $A\left(\Delta, Q_{\infty}\right)$, such that

$$
\left|\mathcal{C}_{1}\left(G_{n}\right)\right| \leq A \log n, \quad \mathbb{P}^{\mathbf{d}^{n}} \text {-a.a.s. }
$$

(c) (Critical regime) Let $\left|Q\left(\mathbf{d}^{n}\right)-2\right| \leq \lambda n^{-\frac{1}{3}}$ for all $n \geq 1$. Then for every $\varepsilon \geq 0$ there exists $A=A(\zeta, \lambda, \varepsilon, \Delta)$ such that for all $n$ large enough

$$
\mathbb{P}^{\mathbf{d}^{n}}\left[A^{-1} n^{\frac{2}{3}} \leq\left|\mathcal{C}_{1}\left(G_{n}\right)\right| \leq A n^{\frac{2}{3}}\right] \geq 1-\varepsilon
$$

(d) (Scaling limit) Let $Q\left(\mathbf{d}^{n}\right)=2+\lambda n^{-\frac{1}{3}}+o\left(n^{-\frac{1}{3}}\right)$ as $n \rightarrow \infty$. Endow $\mathcal{C}_{j}\left(G_{n}\right)$ with the graph distance and the uniform probability measure on its vertices. Then there exists a sequence $\tilde{\mathbf{M}}^{p}(\lambda)=\left(\tilde{M}_{1}^{p}(\lambda), \tilde{M}_{2}^{p}(\lambda), \ldots\right)$ of (random) compact metric measure spaces such that

$$
\frac{1}{n^{1 / 3}}\left(\mathcal{C}_{1}\left(G_{n}\right), \mathcal{C}_{2}\left(G_{n}\right), \ldots\right) \rightarrow \tilde{\mathbf{M}}^{p}(\lambda)
$$

in distribution with respect to the product topology induced by Gromov-HausdorffProkhorov distance on each coordinate.

Proof. (a), (b), (c), (d) follow directly from [JL09, Theorem 2.3], [MR95, Theorem 1], [HM12, Theorem 1.1], [BS20, Theorem 2.4], respectively.

Note that [JL09, Theorem 2.3] and [HM12, Theorem 1.1] consider only degree sequences with $n_{0}\left(\mathbf{d}^{n}\right)=0$. However, it is clear that if $n_{0}\left(\mathbf{d}^{n}\right) \leq \zeta^{\prime} n$ for some $\zeta^{\prime}<1$, the zero-degree vertices have no influence on $Q\left(\mathbf{d}^{n}\right)$ and they just change the constant $A$ in (c). The same holds for the constant $\xi$ in (a).

\subsection{Random interlacements on the Galton-Watson tree}

In this section we consider random interlacements on a particular Galton-Watson tree. These random interlacements will provide a good local description of the vacant set of the configuration model. It will also allow us to specify the distribution of the random degree sequence $\mathbf{d}^{n, u}$ of the vacant graph, and the critical parameter $u^{*}$ of Theorem 4 . For a comprehensive introduction to random interlacements on transient weighted graphs we refer to [Tei09].

The configuration model with a degree sequence satisfying Assumption 1.1 has a locally tree-like nature. More precisely, let $p=\left(p_{i}\right)_{1 \leq i \leq \Delta}$ be a probability mass function as in Assumption 1.1. Consider a rooted random tree $\mathcal{T}$, whose root $\varnothing$ has offspring distribution $p$ and all remaining vertices have offspring distribution $p^{*}=\left(p_{i}^{*}\right)_{0 \leq i \leq \Delta-1}$ defined in (1.7). By Assumption 1.1 and (1.7), $p_{0}^{*}=p_{1}^{*}=0$ and thus, a.s., $\mathcal{T}$ is infinite and the random walk on $\mathcal{T}$ is transient. In [vdH17b, Section 2.1] such random tree is called unimodular Galton-Watson tree with offspring distribution $p^{*}$. We write $\mathbb{P}^{\mathcal{T}}$ for its law and $\mathbb{E}^{\mathcal{T}}$ for the corresponding expectation. It is well known that the configuration model satisfying Assumption 1.1 converges locally to the unimodular Galton-Watson tree $\mathcal{T}$, see e.g. [vdH17b, Theorem 2.11].

We now introduce random interlacements on $\mathcal{T}$. Given a realization of $\mathcal{T}$, let $\bar{Y}=$ $\left(\bar{Y}_{k}\right)_{k \geq 0}$ be the usual (i.e. not lazy) discrete-time simple random walk on $\mathcal{T}$ started at 
$x \in \mathcal{T}$ and $\bar{P}_{x}^{\mathcal{T}}$ its law. Random interlacements on $\mathcal{T}$ at level $u \geq 0$ is defined as a probability distribution $\mathcal{P}_{\mathcal{T}}^{u}$ on the space $\{0,1\}^{\mathcal{T}}$ which samples a random subset $\mathcal{V}_{\mathcal{T}}$ of $\mathcal{T}$, called the vacant set of random interlacements at level $u$, characterized by

$$
\mathcal{P}_{\mathcal{T}}^{u}\left[K \subset \mathcal{V}_{\mathcal{T}}\right]=\exp \left(-u \operatorname{cap}_{\mathcal{T}}(K)\right), \quad \text { for every finite } K \subset \mathcal{T},
$$

where the capacity is given by

$$
\operatorname{cap}_{\mathcal{T}}(K)=\sum_{x \in K} d_{x} \bar{P}_{x}^{\mathcal{T}}\left[\widetilde{H}_{K}^{\bar{Y}}=\infty\right]
$$

$d_{x}$ stands for the degree of $x$ in $\mathcal{T}$, and where $\widetilde{H}_{K}^{\bar{Y}}=\inf \left\{k \geq 1: \bar{Y}_{k} \in K\right\}$ denotes the hitting time of $K$ by the random walk $\bar{Y}$. Let $\mathcal{C}_{\varnothing}$ be the connected component of the root $\varnothing$ in $\mathcal{V}_{\mathcal{T}}$. The critical parameter $u_{\mathcal{T}}^{*}$ of random interlacements on $\mathcal{T}$ is defined as

$$
u_{\mathcal{T}}^{*}=\inf \left\{u \geq 0: \mathcal{P}_{\mathcal{T}}^{u}\left[\left|\mathcal{C}_{\varnothing}\right|=\infty\right]=0\right\} .
$$

Since $\mathcal{T}$ is a random tree, $u_{\mathcal{T}}^{*}$ is, in principle, random. Proposition 2.3 below will show that it is a.s. constant. To state the proposition we need one more definition. Let $\mathcal{T}^{\prime}$ be the usual Galton-Watson tree with offspring distribution $p^{*}$ (including the root vertex $\varnothing^{\prime}$ of $\mathcal{T}^{\prime}$ ). Consider another tree $\tilde{\mathcal{T}}$ obtained from $\mathcal{T}^{\prime}$ by adding an additional vertex $\tilde{\varnothing}$ connected to $\varnothing^{\prime}$. Let

$$
\phi(u)=\mathbb{E}^{\tilde{\mathcal{T}}}\left[\exp \left(-u \bar{P}_{\varnothing^{\prime}}^{\tilde{\mathcal{T}}}\left[H_{\tilde{\varnothing}}^{\bar{Y}}=\infty\right]\right)\right]
$$

where, similarly as above, $\bar{P}_{\varnothing^{\prime}}^{\tilde{\mathcal{T}}}$ denotes the law of the simple random walk $\bar{Y}$ on $\tilde{\mathcal{T}}$ started from $\varnothing^{\prime}$, and $\mathbb{E}^{\tilde{\mathcal{T}}}$ is the expectation over the law of $\tilde{\mathcal{T}}$.

Proposition 2.3. Let $\mathcal{T}$ be the unimodular Galton-Watson tree with offspring distribution $p^{*}$. Then there exists a constant $u^{*} \in(0, \infty)$ such that

$$
u_{\mathcal{T}}^{*}=u^{*}, \quad \mathbb{P}^{\mathcal{T}} \text {-a.s. },
$$

and $u^{*}$ is the unique solution in $(0, \infty)$ of the equation

$$
m_{p}=\sum_{i=1}^{\Delta} p_{i} i(i-1) \phi(u)^{i-2}
$$

Proof. The proof uses the results of [Tas10, Theorem 1], where random interlacements on the (usual) Galton Watson tree were studied in detail.

Let $\mathcal{T}^{\prime}$ be the usual Galton-Watson tree with offspring distribution $p^{*}$, as above. We denote by $\mathcal{T}_{x}$ the subtree of $\mathcal{T}$ containing the vertex $x \in \mathcal{T}$ and all its descendants. Observe that $\mathcal{T}_{x}$ has the same law as $\mathcal{T}^{\prime}$ for every $x \sim \varnothing$. Let $\mathcal{P}_{\mathcal{T}_{x}}^{u}$ be the law of random interlacements at level $u$ on $\mathcal{T}_{x}$ defined analogically to (2.26), and let $\mathcal{C}_{x}$ be the connected component of the vacant set containing $x$. Using [Tas10, Theorem 1] we deduce the existence of a constant $u^{*} \in(0, \infty)$ such that a.s.

$$
u^{*}=\inf \left\{u \geq 0: \mathcal{P}_{\mathcal{T}^{\prime}}^{u}\left[\left|\mathcal{C}_{\varnothing^{\prime}}\right|=\infty\right]=0\right\}=\inf \left\{u \geq 0: \mathcal{P}_{\mathcal{T}_{x}}^{u}\left[\left|\mathcal{C}_{x}\right|=\infty\right]=0\right\}
$$

We now show (2.30). Since $\mathcal{P}_{\mathcal{T}}^{u}\left[\varnothing \in \mathcal{V}_{\mathcal{T}}\right]=e^{-u \operatorname{cap}_{\mathcal{T}}(\varnothing)}>0$ for every $u \geq 0, \mathbb{P}^{\mathcal{T}}$-a.s., we see that

$$
\mathcal{P}_{\mathcal{T}}^{u}\left[\left|\mathcal{C}_{\varnothing}\right|=\infty\right]=0 \quad \text { iff } \quad \mathcal{P}_{\mathcal{T}}^{u}\left[\left|\mathcal{C}_{\varnothing}\right|=\infty \mid \varnothing \in \mathcal{V}_{\mathcal{T}}\right]=0, \quad \mathbb{P}^{\mathcal{T}} \text {-a.s. }
$$


On the other hand, since the events $\left\{\left|\mathcal{C}_{\varnothing} \cap \mathcal{T}_{x}\right|=\infty\right\}$ are conditionally independent under $\mathcal{P}_{\mathcal{T}}^{u}$ given $\varnothing \in \mathcal{V}_{\mathcal{T}}$, as can be deduced e.g. from [Tei09, Theorem 5.1],

$$
\begin{aligned}
\mathcal{P}_{\mathcal{T}}^{u} & {\left[\left|\mathcal{C}_{\varnothing}\right|=\infty \mid \varnothing \in \mathcal{V}_{\mathcal{T}}\right]=\mathcal{P}_{\mathcal{T}}^{u}\left[\exists x \sim \varnothing:\left|\mathcal{C}_{\varnothing} \cap \mathcal{T}_{x}\right|=\infty \mid \varnothing \in \mathcal{V}_{\mathcal{T}}\right] } \\
& =1-\prod_{x \sim \varnothing}\left(1-\mathcal{P}_{\mathcal{T}}^{u}\left[\left|\mathcal{C}_{\varnothing} \cap \mathcal{T}_{x}\right|=\infty \mid \varnothing \in \mathcal{V}_{\mathcal{T}}\right]\right) \\
& =1-\prod_{x \sim \varnothing}\left(1-\mathcal{P}_{\mathcal{T}}^{u}\left[\left|\mathcal{C}_{\varnothing} \cap \mathcal{T}_{x}\right|=\infty \mid \varnothing, x \in \mathcal{V}_{\mathcal{T}}\right] \mathcal{P}_{\mathcal{T}}^{u}\left[x \in \mathcal{V}_{\mathcal{T}} \mid \varnothing \in \mathcal{V}_{\mathcal{T}}\right]\right) .
\end{aligned}
$$

By [Tas10, (3.8),(3.9)],

$$
\mathcal{P}_{\mathcal{T}}^{u}\left[\left|\mathcal{C}_{\varnothing} \cap \mathcal{T}_{x}\right|=\infty \mid \varnothing, x \in \mathcal{V}_{\mathcal{T}}\right]=\mathcal{P}_{\mathcal{T}_{x}}^{u}\left[\left|\mathcal{C}_{x}\right|=\infty \mid x \in \mathcal{V}_{\mathcal{T}_{x}}\right]
$$

Finally, since $\mathcal{P}_{\mathcal{T}}^{u}\left[x \in \mathcal{V}_{\mathcal{T}} \mid \varnothing \in \mathcal{V}_{\mathcal{T}}\right]>0$ a.s., we deduce from (2.33)-(2.35) that a.s.

$$
u_{\mathcal{T}}^{*}=\inf \left\{u \geq 0: \mathcal{P}_{\mathcal{T}}^{u}\left[\left|\mathcal{C}_{\varnothing}\right|=\infty\right]=0\right\}=\inf \left\{u \geq 0: \mathcal{P}_{\mathcal{T}_{x}}^{u}\left[\left|\mathcal{C}_{x}\right|=\infty\right]=0\right\}=u^{*},
$$

by (2.32), proving (2.30).

To show (2.31), note that by [Tas10, Theorem 1], $u^{*}$ is the unique solution of the equation

$$
\left(f^{-1}\right)^{\prime}\left(\mathbb{E}^{\mathcal{T}^{\prime}}\left[\exp \left(-u \operatorname{cap}_{\mathcal{T}^{\prime}}\left(\varnothing^{\prime}\right)\right)\right]\right)=1,
$$

where $f$ is the probability generating function of $p^{*}$. Let $x$ be an arbitrary neighbour of the root $\varnothing^{\prime}$. By (2.27) and the Markov property,

$$
\begin{aligned}
\mathbb{E}^{\mathcal{T}^{\prime}}\left[\exp \left(-u \operatorname{cap}_{\mathcal{T}^{\prime}}\left(\varnothing^{\prime}\right)\right)\right] & =\mathbb{E}^{\mathcal{T}^{\prime}}\left[\mathbb{E}^{\mathcal{T}^{\prime}}\left[\exp \left(-u d_{\varnothing^{\prime}} \sum_{y \sim \varnothing^{\prime}} \frac{1}{d_{\varnothing^{\prime}}} \bar{P}_{y}^{\mathcal{T}^{\prime}}\left[\widetilde{H}_{\varnothing^{\prime}}^{\bar{Y}}=\infty\right]\right) \mid d_{\varnothing^{\prime}}\right]\right] \\
& =\mathbb{E}^{\mathcal{T}^{\prime}}\left[\prod_{y \sim \varnothing^{\prime}} \mathbb{E}^{\mathcal{T}^{\prime}}\left[\exp \left(-u \bar{P}_{y}^{\mathcal{T}^{\prime}}\left[\widetilde{H}_{\varnothing^{\prime}}^{\bar{Y}}=\infty\right]\right) \mid d_{\varnothing^{\prime}}\right]\right] \\
& =\mathbb{E}^{\mathcal{T}^{\prime}}\left[\phi(u)^{d_{\varnothing^{\prime}}}\right]=f(\phi(u)),
\end{aligned}
$$

where, in the second equality we used that $\bar{P}_{y}^{\mathcal{T}^{\prime}}\left[\widetilde{H}_{\varnothing^{\prime}}^{\bar{Y}}=\infty\right]$ for $y \sim \varnothing^{\prime}$ are i.i.d. under $\mathbb{P}^{\mathcal{T}^{\prime}}\left[\cdot \mid d_{\varnothing^{\prime}}\right]$, and in the third equality we used the equality

$$
\mathbb{E}^{\mathcal{T}^{\prime}}\left[\exp \left(-u \bar{P}_{y}^{\mathcal{T}^{\prime}}\left[\widetilde{H}_{\varnothing^{\prime}}^{\bar{Y}}=\infty\right]\right) \mid d_{\varnothing^{\prime}}\right]=\phi(u),
$$

which follows from the fact that the probability on the left-hand side depends only on the shape of $\mathcal{T}_{y} \cup\left\{\varnothing^{\prime}\right\}$, which has the same law as the shape of the tree $\tilde{\mathcal{T}}$ introduced above (2.29). Using $\left(f^{-1}\right)^{\prime}=1 /\left(f^{\prime} \circ f^{-1}\right)$ and (2.38), shows that (2.37) is equivalent with $f^{\prime}(\phi(u))=1$. Together with definition (1.7) of $p^{*}$, this finishes the proof of (2.31).

\section{Random walk estimates}

The aim of this section is to estimate the probability that the random walk $X$ does not visit certain subsets of $B(x, 1)$ before time $2 u m_{p} n$ (see Proposition 3.1). Those estimates will later be used to describe the distribution of $\mathbf{d}^{n, u}$, the random degree sequence of the vacant graph.

For a vertex $x \in[n]$, we use $x_{i} \sim x, i \in\left[d_{x}^{n}\right]$, to denote its neighbours listed in the increasing order. For any $A \subset\left[d_{x}^{n}\right]$, let

$$
B_{x, A}=\{x\} \cup\left\{x_{i}: i \in A\right\} .
$$


Observe that if $B(x, 1) \subset G_{n}$ is a tree, then

$$
\begin{aligned}
P^{G_{n}}\left[d_{x}^{n, u}=i\right]=\sum_{\substack{C \subset\left[d_{x}^{n}\right] \\
|C|=i}} P^{G_{n}}\left[\mathcal{V}_{G_{n}}^{u} \cap B(x, 1)=B_{x, C}\right] \\
=\sum_{\substack{C \subset\left[d_{x}^{n}\right] \\
|C|=i}} P^{G_{n}}\left[\left\{H_{B_{x, C}}>2 u m_{p} n\right\} \backslash\left(\bigcup_{y \in B(x, 1) \backslash B_{x, C}}\left\{H_{y}>2 u m_{p} n\right\}\right)\right] \\
=\sum_{\substack{C \subset\left[d_{x}^{n}\right] \\
|C|=i}}\left(P^{G_{n}}\left[H_{B_{x, C}}>2 u m_{p} n\right]-P^{G_{n}}\left[\bigcup_{y \in B(x, 1) \backslash B_{x, C}}\left\{H_{B_{x, C} \cup\{y\}}>2 u m_{p} n\right\}\right]\right) \\
=\sum_{\substack{C \subset\left[d_{x}^{n}\right] \\
|C|=i}} \sum_{\substack{A \subset\left[d_{x}^{n}\right] \\
A \supset C}}(-1)^{|A|-|C|} P^{G_{n}}\left[H_{B_{x, A}}>2 u m_{p} n\right],
\end{aligned}
$$

where for the last identity we used the inclusion-exclusion formula. Thus, estimates on the probability on the right-hand side of (3.2) enable us to control the distribution of the degree of $x$ in the vacant graph.

These estimates are shown for deterministic graphs that are "typical" under Assumption 1.1, that is on the set

$$
\mathcal{G}_{\text {typ }}^{\mathbf{d}^{n}}=\left\{G_{n} \in \mathcal{G}^{\mathbf{d}^{n}}: \lambda_{G_{n}} \geq c \log ^{-l} n\right\}
$$

where $c>0$ and $l \geq 0$ are as in Assumption 1.1. In particular, every $G_{n} \in \mathcal{G}_{\text {typ }}^{\mathbf{d}^{n}}$ is connected. We recall that we always assume that $\mathbf{d}^{n}$ satisfies Assumption 1.1.

Proposition 3.1. For every $\kappa>0$ there is $c \in(0, \infty)$ such that for every $n \geq 2$, $G_{n} \in \mathcal{G}_{\text {typ }}^{\mathbf{d}^{n}}, x \in[n], A \subset\left[d_{x}^{n}\right]$, with $t_{n}=\log ^{3 l+3} n$,

$$
\left|P^{G_{n}}\left[H_{B_{x, A}}>\kappa n\right]-\exp \left(-\frac{\kappa n}{L_{n}} \sum_{y \in B_{x, A}} d_{y}^{n} P_{y}^{G_{n}}\left[\widetilde{H}_{B_{x, A}}>t_{n}\right]\right)\right| \leq \frac{c t_{n}}{n} .
$$

To prove Proposition 3.1 we need several preparatory lemmas. To simplify the notation, we set $B=B_{x, A}$, define $\mathcal{C}_{1}\left(G_{n} \backslash B\right)$ to be the largest connected component of $G_{n} \backslash B$, and $S=B \cup \mathcal{C}_{1}\left(G_{n} \backslash B\right)$ (which, according to our convention, we identify with the corresponding induced subgraph of $G_{n}$ ). The reason to introduce this subgraph is that in order to understand the behaviour of $H_{B}$ under $P^{G_{n}}$, we need to study the random walk on $G_{n} \backslash B$ which might not be connected, unlike $S$. The two next lemmas show that replacing $G_{n} \backslash B$ by $S$ does not make much difference.

We denote $P^{S}$ the law of the lazy discrete time random walk $X$ on $S$, started from its (unique) stationary distribution $\pi^{S}$, and write $E^{S}$ for the corresponding expectation.

Lemma 3.2. There are $c, c^{\prime} \in(0, \infty)$ such that for every $n \geq 2, G_{n} \in \mathcal{G}_{\text {typ }}^{\mathbf{d}^{n}}, x \in[n]$, and every $B$ such that $x \in B \subset B(x, 1)$,

$$
\begin{aligned}
& \left|S^{c}\right| \leq c \log ^{l} n, \quad \text { where } S^{c}=G_{n} \backslash S, \\
& \sup _{y \in S^{c}} E_{y}^{G_{n}}\left[H_{B}\right] \leq c \log ^{3 l} n, \\
& E^{S}\left[H_{B}\right] \geq c^{\prime} n .
\end{aligned}
$$

Proof. We start with the proof of (3.5). Let $h\left(G_{n}\right)$ be the isoperimetric constant of $G_{n}$ defined in (2.8). By Assumption 1.1, we know that

$$
\frac{1}{\Delta n} \leq \pi^{G_{n}}(x) \leq \frac{\Delta}{n}
$$


The Cheeger inequality (2.9) then implies that

$$
\lambda_{G_{n}} \leq \operatorname{ch}\left(G_{n}\right)=c \min _{A \subset[n], \pi^{G_{n}}(A) \leq \frac{1}{2}}\left\{\frac{Q(A)}{\pi^{G_{n}}(A)}\right\} \leq \frac{c \Delta^{2}}{2} \min _{A \subset[n], \pi^{G_{n}}(A) \leq \frac{1}{2}}\left\{\frac{\left|\partial_{e} A\right|}{|A|}\right\} .
$$

Since $G_{n} \in \mathcal{G}_{\text {typ }}^{\mathbf{d}^{n}}$ is connected, the graph $G_{n} \backslash B$ has at most $\left|\partial_{e} B\right| \leq \Delta^{2}$ components and at least $n-|B| \geq n-(\Delta+1)$ vertices. Therefore

$$
\left|\mathcal{C}_{1}\left(G_{n} \backslash B\right)\right| \geq \frac{n-(\Delta+1)}{\Delta^{2}} .
$$

If, in addition, we assume that $\pi^{G_{n}}\left(\mathcal{C}_{1}(G \backslash B)\right) \leq \frac{1}{2}$, then by (3.9), since $\partial_{e} \mathcal{C}_{1}\left(G_{n} \backslash B\right) \subset$ $\partial_{e} B$

$$
\lambda_{G_{n}} \leq \frac{c \Delta^{2}}{2} \frac{\partial_{e} \mathcal{C}_{1}\left(G_{n} \backslash B\right) \mid}{\mathcal{C}_{1}\left(G_{n} \backslash B\right)} \leq \frac{c \Delta^{6}}{2(n-(\Delta+1))},
$$

which is a contradiction with $G_{n} \in \mathcal{G}_{\text {typ }}^{\mathbf{d}^{n}}$. Therefore, $\pi^{G_{n}}\left(S^{c}\right) \leq 1-\pi^{G_{n}}\left(\mathcal{C}_{1}(G \backslash B)\right)<\frac{1}{2}$. Using $\partial_{e} S^{c} \subset \partial_{e} B$ and (3.9), we obtain

$$
\lambda_{G_{n}} \leq \frac{c \Delta^{2}}{2} \frac{\left|\partial_{e} S^{c}\right|}{\left|S^{c}\right|} \leq \frac{c \Delta^{4}}{2\left|S^{c}\right|}
$$

and (3.5) follows, since $G_{n} \in \mathcal{G}_{\text {typ }}^{\mathrm{d}^{n}}$.

To show (3.6), first note that the expected cover time $C_{G}$ of any connected graph $G_{n}=(V, E)$ is bounded by $c|V|^{3}$ for some universal constant $c$ (see e.g. [AF02, Theorem 6.1]). For any $x \in S^{c}$, let $S_{x}^{c}$ be the connected component of $S^{c}$ containing $x$. By (3.5),

$$
\sup _{x \in S^{c}} E_{x}^{G_{n}}\left[H_{B}\right] \leq \sup _{x \in S^{c}} C_{S_{x}^{c} \cup B} \leq c\left|S^{c} \cup B\right|^{3} \leq c \log ^{3 l} n .
$$

For the proof of (3.7), note that by [AB92, Lemma 2], $E^{G_{n}}\left[H_{B}\right] \geq c^{\prime \prime} n$, for some $c^{\prime \prime}>0$. In addition, the stationary measure $\pi^{S}$ on $S$ satisfies $\pi^{G_{n}}(x) \leq \pi^{S}(x)$ for every $x \in S \backslash B$, since the degrees of $x$ in $G_{n}$ and in $S$ are the same but $S \subset G_{n}$. Hence, using (3.6), we get

$$
\begin{aligned}
c^{\prime \prime} n \leq E^{G_{n}}\left[H_{B}\right] & =\sum_{x \in S} \pi^{G_{n}}(x) E_{x}^{G_{n}}\left[H_{B}\right]+\sum_{x \in S^{c}} \pi^{G_{n}}(x) E_{x}^{G_{n}}\left[H_{B}\right] \\
& \leq E^{S}\left[H_{B}\right]+\frac{\Delta \log ^{4 l} n}{n}
\end{aligned}
$$

and (3.7) follows.

Lemma 3.3. There is $c<\infty$ such that for every $n \geq 2, G_{n} \in \mathcal{G}_{\text {typ }}^{\mathbf{d}^{n}}, x \in[n]$, and every $B$ such that $x \in B \subset B(x, 1)$,

$$
\operatorname{diam}(S \backslash B) \leq c \log ^{l+1} n .
$$

Proof. It is well known (see e.g. [LPW09, Theorem 12.3 and (7.3)]), that one can use the spectral gap to bound the diameter of connected graphs. Using in addition that $G_{n} \in \mathcal{G}_{\text {typ }}^{\mathrm{d}^{n}}$, we have

$$
\operatorname{diam}\left(G_{n}\right) \leq 2 \log \left(\frac{4}{\min _{x \in[n]} \pi^{G_{n}}(x)}\right) \lambda_{G_{n}}^{-1} \leq c \log ^{l+1} n .
$$

We now claim that

$$
\operatorname{diam}(S) \leq \operatorname{diam}\left(G_{n}\right)
$$


Indeed, let $\gamma^{G_{n}}(x, y)$ for a geodesic path between $x \in[n]$ and $y \in[n]$ in $G_{n}$ (that is a path whose length is $\left.\operatorname{dist}_{G_{n}}(x, y)\right)$. Let $x, y \in S$. If $\gamma^{G_{n}}(x, y) \cap S^{c}=\emptyset$, then $\operatorname{dist}_{S}(x, y)=\operatorname{dist}_{G_{n}}(x, y)$. On the other hand, if $\gamma^{G_{n}}(x, y)$ intersects $S^{c}$, then it must pass through at least two different edges whose one vertex is in $B$ and one in $S^{c}$. Since $\operatorname{diam}(B) \leq 2$, we can connect the endpoints in $B$ of those two edges by a path of length at most two lying in $B$, and construct a path $\tilde{\gamma}^{G_{n}}(x, y) \subset S$ of length at most $\operatorname{dist}_{G_{n}}(x, y)$ connecting $x, y$. Hence, $\operatorname{dist}_{G_{n}}(x, y)=\operatorname{dist}_{S}(x, y)$ again and (3.17) follows.

Finally, we claim that

$$
\operatorname{diam}(S \backslash B) \leq 4 \Delta^{2} \operatorname{diam}\left(G_{n}\right) .
$$

Assume by contradiction that (3.18) does not hold. Then there are $x, y \in S \backslash B$ with $\operatorname{dist}_{S \backslash B}(x, y)>4 \Delta^{2} \operatorname{diam}\left(G_{n}\right)$. Let $\gamma=\left(x=z_{0}, z_{1}, \ldots, z_{M}=y\right)$ be a geodesic in $S \backslash B$ linking those two points, $M>4 \Delta^{2} \operatorname{diam}\left(G_{n}\right)$. Set $m=3 \operatorname{diam}\left(G_{n}\right)$ and $\ell=M / m \geq$ $4 \Delta^{2} / 3$. Since $\gamma$ is a geodesic path in $S \backslash B$,

$$
\operatorname{dist}_{S \backslash B}\left(z_{i m}, z_{j m}\right)=3|i-j| \operatorname{diam}\left(G_{n}\right), \quad \text { for every } 0 \leq i, j \leq \ell .
$$

On the other hand, by $(3.17), \operatorname{dist}_{S}\left(z_{(i-1) m}, z_{i m}\right) \leq \operatorname{diam}\left(G_{n}\right)$ for $1 \leq i \leq \ell$. Therefore, there is a geodesic path $\gamma^{i}$ in $S$ linking $z_{(i-1) m}, z_{i m}$ of length at most $\operatorname{diam}\left(G_{n}\right)$. Every $\gamma^{i}$ has to intersect $B$, due to (3.19). Let $a_{i}$ be the first point on $\gamma^{i}$ contained in $\partial_{S} B=$ $\left\{y \in S: \operatorname{dist}_{G_{n}}(y, B)=1\right\}$. Since $\ell \geq 4 \Delta^{2} / 3$ and $\left|\partial_{S} B\right| \leq \Delta^{2}$, by Assumption 1.1, there exist $1 \leq i<j \leq \ell$ such that $a_{i}=a_{j}$. By considering the part of $\gamma^{i}$ between $z_{i}$ and $a_{i}$ and the part of $\gamma^{j}$ between $z_{j}$ and $a_{j}=a_{i}$, we see that there is a path in $S \backslash B$ linking $z_{i}$ and $z_{j}$ of length at most $2 \operatorname{diam}\left(G_{n}\right)$, leading to contradiction with (3.19), and proving (3.18).

For the next lemma, let $\sigma_{B}$ be the quasi-stationary distribution of the random walk on $S$ conditioned on not hitting $B$, uniquely determined by (recall that $S \backslash B=\mathcal{C}_{1}\left(G_{n} \backslash B\right.$ ) is connected)

$$
P_{\sigma_{B}}^{S}\left[X_{1}=y \mid H_{B}>1\right]=\sigma_{B}(y) .
$$

We need the following estimate on the convergence rate of the conditioned random walk on $S$ towards $\sigma_{B}$.

Lemma 3.4. There is $c \in(0, \infty)$ such that for every $n \geq 2, G_{n} \in \mathcal{G}_{\text {typ }}^{\mathbf{d}^{n}}, x \in[n]$ and every $B$ such that $x \in B \subset B(x, 1)$, with $t_{n}^{\prime} \geq \log ^{2 l+2} n$,

$$
\sup _{z, y \in S \backslash B}\left|P_{z}^{S}\left[X_{t_{n}^{\prime}}=y \mid H_{B}>t_{n}^{\prime}\right]-\sigma_{B}(y)\right| \leq c \exp \left(-\frac{c t_{n}^{\prime}}{\log ^{l} n}\right) .
$$

Proof. Let $0<\lambda_{1}^{B}<\lambda_{2}^{B} \leq \cdots \leq \lambda_{|S \backslash B|}^{B}$ be the eigenvalues of the generator of the random walk on $S$ killed on hitting $B$. By [C̈T13, Lemma A.2], the claim of the lemma follows, if we can show that

$$
e^{-t_{n}^{\prime}\left(\lambda_{2}^{B}-\lambda_{1}^{B}\right)}|S \backslash B|\left(\sup _{x \in S \backslash B} \frac{\sigma_{B}(x)}{\pi^{S}(x)^{\frac{1}{2}}}\right)^{2}\left(\inf _{x \in S \backslash B} \frac{\sigma_{B}(x)}{\pi^{S}(x)^{\frac{1}{2}}}\right)^{-1} \leq c \exp \left(-\frac{c t_{n}^{\prime}}{\log ^{l} n}\right) .
$$

Hence, we need to provide lower bounds for $\lambda_{2}^{B}-\lambda_{1}^{B}$ and $\inf _{x \in S \backslash B} \sigma_{B}(x)$.

We start with $\lambda_{2}^{B}-\lambda_{1}^{B}$. By the eigenvalue interlacing inequality (see e.g. [Hae95, Corollary 2.2]) we have $\lambda_{2}^{B} \geq \lambda_{G_{n}}$. On the other hand, by [AB92, Lemma 2 and the paragraph following equation $(12)], \lambda_{1}^{B}=E_{\sigma_{B}}^{S}\left[H_{B}\right]^{-1} \leq E^{S}\left[H_{B}\right]^{-1}$. Since $G_{n} \in \mathcal{G}_{\text {typ }}^{\mathbf{d}^{n}}$, by (3.7),

$$
\lambda_{B}^{2}-\lambda_{B}^{1} \geq \lambda_{G_{n}}-\frac{1}{E^{S}\left[H_{B}\right]} \geq \frac{c}{\log ^{l} n} .
$$


We now estimate $\inf _{x \in S \backslash B} \sigma_{B}(x)$. Let $x \in S \backslash B$ and $P_{x}^{S}$ be the law of the random walk $X$ on $S$ started in $x$. By reversibility, for all $x^{\prime} \in S \backslash B$ and $k \geq 0$,

$$
\pi^{G_{n}}\left(x^{\prime}\right) P_{x^{\prime}}^{S}\left[X_{k}=x \mid H_{B}>k\right]=\pi^{G_{n}}(x) P_{x}^{S}\left[X_{k}=x^{\prime} \mid H_{B}>k\right] \frac{P_{x}^{S}\left[H_{B}>k\right]}{P_{x^{\prime}}^{S}\left[H_{B}>k\right]} .
$$

In order to bound the above ratio, note that

$$
P_{x}^{S}\left[H_{B}>k\right] \geq P_{x}^{S}\left[H_{x^{\prime}}<H_{B}, H_{B}>k\right] \geq P_{x}^{S}\left[H_{x^{\prime}}<H_{B}\right] P_{x^{\prime}}^{S}\left[H_{B}>k\right] .
$$

Further, by Lemma 3.3, $\operatorname{diam}(S \backslash B) \leq c \log ^{l+1} n$. Thus we can find a path of length at most $c \log ^{l+1} n$, connecting $x$ and $x^{\prime}$ and not passing through $B$. By letting the random walk to follow this path, using $p_{x y} \geq 1 /(2 \Delta)$ if $x \sim y$ by (2.3), this yields

$$
P_{x}^{S}\left[H_{x^{\prime}}<H_{B}\right] \geq(2 \Delta)^{-c \log ^{l+1} n} .
$$

Finally, by, standard properties of the quasi-stationary distribution, (see e.g. [ČT13, Lemma A.2.]), $\lim _{k \rightarrow \infty} P_{x}^{S}\left[X_{k}=x^{\prime} \mid H_{B}>k\right]=\sigma_{B}\left(x^{\prime}\right)$ uniformly for all $x, x^{\prime} \in S \backslash B$. Therefore, taking the limit $k \rightarrow \infty$ in (3.24), using (3.8), (3.25) and (3.26), for some $c, c^{\prime} \in(0, \infty)$,

$$
\sigma_{B}(x) \geq c^{\prime} \sigma_{B}\left(x^{\prime}\right) \Delta^{-c \log ^{l+1} n}, \quad \text { for all } x, x^{\prime} \in S \backslash B .
$$

Summing this inequality over all $x^{\prime} \in S \backslash B$, using the fact that $\sigma_{B}$ is a probability measure supported on $S \backslash B$, we obtain

$$
\sigma_{B}(x) \geq|S \backslash B|^{-1} \Delta^{-c \log ^{l+1} n} \geq \exp \left(-c^{\prime} \log ^{l+1} n\right), \quad \text { for all } x \in S \backslash B .
$$

After inserting (3.8), (3.23) and (3.28) into the left-hand side of (3.22), using $t_{n}^{\prime} \geq$ $\log ^{2 l+2} n$, we obtain

$$
\begin{aligned}
e^{-t_{n}^{\prime}\left(\lambda_{B}^{2}-\lambda_{B}^{1}\right)}|S \backslash B|\left(\sup _{x \in S \backslash B} \frac{\sigma_{B}(x)}{\pi^{S}(x)^{\frac{1}{2}}}\right)^{2}\left(\inf _{x \in S \backslash B} \frac{\sigma_{B}(x)}{\pi^{S}(x)^{\frac{1}{2}}}\right)^{-1} \\
\leq c n^{\frac{3}{2}} \exp \left(-\frac{c^{\prime} t_{n}^{\prime}}{\log ^{l} n}+c^{\prime \prime} \log ^{l+1} n\right) \leq c \exp \left(-\frac{c^{\prime} t_{n}^{\prime}}{\log ^{l} n}\right) .
\end{aligned}
$$

This shows (3.22) and completes the proof.

Lemma 3.5. For every $\kappa>0$ there is $c \in(0, \infty)$ such that for every $n \geq 2, G_{n} \in \mathcal{G}_{\text {typ }}^{\mathbf{d}^{n}}$, $x \in[n]$, and every $B$ such that $x \in B \subset B(x, 1)$, with $t_{n}=\log ^{3 l+3} n$,

$$
\left|P^{G_{n}}\left[H_{B}>\kappa n\right]-\exp \left(-\frac{\kappa n}{E_{\sigma_{B}}^{S}\left[H_{B}\right]}\right)\right| \leq \frac{c t_{n}}{n} .
$$

Proof. Recall that $\pi^{S}$ denotes the stationary measure of the random walk on $S$. Then by Lemma 3.2, for $x \in S \backslash B$, since $d_{x}^{n}$ agrees with the degree $d_{x}^{S}$ of $x$ in $S$,

$$
\pi^{G_{n}}(x) \leq \pi^{S}(x)=\frac{d_{x}^{n}}{\sum_{y \in S} d_{y}^{S}} \leq \frac{d_{x}^{n}}{\sum_{y \in[n]} d_{y}^{n}-c \log ^{l} n} \leq \pi^{G_{n}}(x)\left(1+\frac{c \log ^{l} n}{n}\right) .
$$

Using the estimate (3.5) on the size of $S^{c}$ and the fact that $\pi^{G_{n}}(x) \leq c n^{-1}$, we obtain

$$
\begin{aligned}
P^{G_{n}}\left[H_{B}>\kappa n\right] & =\sum_{x \in S} \pi^{G_{n}}(x) P_{x}^{G_{n}}\left[H_{B}>\kappa n\right]+\sum_{x \in S^{c}} \pi^{G_{n}}(x) P_{x}^{G_{n}}\left[H_{B}>\kappa n\right] \\
& =P^{S}\left[H_{B}>\kappa n\right]+O\left(\frac{\log ^{l} n}{n}\right) .
\end{aligned}
$$


Using the Markov property and Lemma 3.4, for $G_{n} \in \mathcal{G}_{\text {typ }}^{\mathbf{d}^{n}}$ and some $c>0$,

$$
\begin{aligned}
& \left|P^{S}\left[H_{B}>\kappa n\right]-\exp \left(-\frac{\kappa n}{E_{\sigma_{B}}^{S}\left[H_{B}\right]}\right)\right| \\
& =\left|P^{S}\left[H_{B}>t_{n}\right] E^{S}\left[P_{X_{t_{n}}}^{S}\left[H_{B}>\kappa n-t_{n}\right] \mid H_{B}>t_{n}\right]-\exp \left(-\frac{\kappa n}{E_{\sigma_{B}}^{S}\left[H_{B}\right]}\right)\right| \\
& \leq\left|P^{S}\left[H_{B}>t_{n}\right] P_{\sigma_{B}}^{S}\left[H_{B}>\kappa n-t_{n}\right]-\exp \left(-\frac{\kappa n}{E_{\sigma_{B}}^{S}\left[H_{B}\right]}\right)\right|+c \exp \left(\frac{-c^{\prime} t_{n}}{\log ^{l} n}\right) .
\end{aligned}
$$

By, e.g., [AF02, (3.82) and below], the hitting time $H_{B}$ under $P_{\sigma_{B}}^{S}$ has geometric distribution with mean $E_{\sigma_{B}}^{S}\left[H_{B}\right]$. Moreover, by the same arguments as above (3.23) and by (3.7), $E_{\sigma_{B}}^{S}\left[H_{B}\right] \geq E^{S}\left[H_{B}\right] \geq$ cn. Thus,

$$
\begin{aligned}
P_{\sigma_{B}}^{S}\left[H_{B}>\kappa n-t_{n}\right] & =\left(1-\frac{1}{E_{\sigma_{B}}^{S}\left[H_{B}\right]}\right)^{\kappa n-t_{n}} \\
& =\exp \left(-\frac{\kappa n}{E_{\sigma_{B}}^{S}\left[H_{B}\right]}\right)+O\left(\frac{t_{n}}{n}\right) .
\end{aligned}
$$

Applying [AB92, Theorem 3],

$$
P^{S}\left[H_{B} \leq t_{n}\right] \leq 1-\left(1-\frac{1}{\lambda_{S} E_{\sigma_{B}}^{S}\left[H_{B}\right]}\right) \exp \left(-\frac{t_{n}}{E_{\sigma_{B}}^{S}\left[H_{B}\right]}\right) \leq \frac{c t_{n}}{n} .
$$

Combining (3.34) and (3.35), one shows easily that the right-hand side of (3.33) is bounded by $c t_{n} / n$. Together with (3.32) this then yields the claim of the lemma.

The final ingredient for the proof of Proposition 3.1 is the next estimate on $E_{\sigma_{B}}^{S}\left[H_{B}\right]$.

Lemma 3.6. There is $c \in(0, \infty)$ such that for every $n \geq 2, G_{n} \in \mathcal{G}_{\text {typ }}^{\mathbf{d}^{n}}, x \in[n]$ and $x \in B \subset B(x, 1)$, with $t_{n}=\log ^{3 l+3} n$,

$$
\left|\frac{1}{E_{\sigma_{B}}^{S}\left[H_{B}\right]}-\sum_{y \in B_{x}} \frac{d_{y}^{n}}{L_{n}} P_{y}^{G_{n}}\left[\widetilde{H}_{B}>t_{n}\right]\right| \leq \frac{c t_{n}}{n^{2}} .
$$

Proof. Let $d_{B}^{n}=\sum_{x \in B} d_{x}^{n}$ and $\pi_{B}(x)=\frac{d_{x}^{n}}{d_{B}^{n}}$ for $x \in B$, and $\pi_{B}(x)=0$ otherwise.

We first claim that

$$
1=\pi^{G_{n}}(B) E_{\pi_{B}}^{G_{n}}\left[\widetilde{H}_{B}\right] .
$$

Recall that $P^{G_{n}}$ is the law of the stationary lazy random walk on $G_{n}$. Therefore,

$$
\begin{aligned}
P^{G_{n}}\left[\widetilde{H}_{B}=k\right] & =P^{G_{n}}\left[\widetilde{H}_{B} \geq k\right]-P^{G_{n}}\left[\widetilde{H}_{B} \geq k+1\right] \\
& =P^{G_{n}}\left[\widetilde{H}_{B} \geq k\right]-P^{G_{n}}\left[H_{B} \geq k\right] \\
& =\pi^{G_{n}}(B) P_{\pi_{B}}^{G_{n}}\left[\widetilde{H}_{B} \geq k\right] .
\end{aligned}
$$

Summing over $k \geq 1$, proves (3.37).

Observe that by $(3.7), E_{\pi_{B}}^{G_{n}}\left[\widetilde{H}_{B} \mathbf{1}_{\left\{X_{1} \in S^{c}\right\}}\right] \leq \sup _{x \in S^{c}} E_{x}^{G_{n}}\left[H_{B}\right] \leq c \log ^{3 l} n$. Using this in the third step, we obtain

$$
\begin{aligned}
& \frac{1}{\pi^{G_{n}}(B)}=E_{\pi_{B}}^{G_{n}}\left[\widetilde{H}_{B}\right] \\
& =E_{\pi_{B}}^{G_{n}}\left[\widetilde{H}_{B} \mathbf{1}_{\left\{X_{1} \in S^{c}\right\}}\right]+E_{\pi_{B}}^{G_{n}}\left[\widetilde{H}_{B} \mathbf{1}_{\left\{X_{1} \in S \cap \tilde{H}_{B} \leq t_{n}\right\}}\right]+E_{\pi_{B}}^{G_{n}}\left[\widetilde{H}_{B} \mathbf{1}_{\left\{X_{1} \in S \cap \tilde{H}_{B}>t_{n}\right\}}\right] \\
& =O\left(t_{n}\right)+P_{\pi_{B}}^{G_{n}}\left[\widetilde{H}_{B}>t_{n}\right] P_{\pi_{B}}^{G_{n}}\left[X_{1} \in S \mid \widetilde{H}_{B}>t_{n}\right] E_{\pi_{B}}^{G_{n}}\left[\widetilde{H}_{B} \mid X_{1} \in S, \widetilde{H}_{B} \geq t_{n}\right] .
\end{aligned}
$$


For $S^{c} \neq \varnothing$, by the Markov inequality and (3.6),

$$
P_{\pi_{B}}^{G_{n}}\left[\widetilde{H}_{B}>\log ^{3 l+1} n \mid X_{1} \in S^{c}\right] \leq \sup _{x \in S^{c}} P^{G_{n}}\left[H_{B}>\log ^{3 l+1} n\right] \leq \log ^{-1} n .
$$

By iterating this $\log ^{2} n$ times, and using that the walk does not leave $S^{c}$, we obtain $P_{\pi_{B}}^{G_{n}}\left[\widetilde{H}_{B}>\log ^{3 l+3} n \mid X_{1} \in S^{c}\right] \leq \exp \left(-\log ^{2} n\right)$. Therefore,

$$
\begin{aligned}
P_{\pi_{B}}^{G_{n}}\left[X_{1} \in S \mid \widetilde{H}_{B}>t_{n}\right] & =1-\frac{P_{\pi_{B}}^{G_{n}}\left[X_{1} \in S^{c}\right]}{P_{\pi_{B}}^{G_{n}}\left[\widetilde{H}_{B}>t_{n}\right]} P_{\pi_{B}}^{G_{n}}\left[\widetilde{H}_{B}>t_{n} \mid X_{1} \in S^{c}\right] \\
& \geq 1-\frac{P_{\pi_{B}}^{G_{n}}\left[X_{1} \in S^{c}\right]}{P_{\pi_{B}}^{G_{n}}\left[\widetilde{H}_{B}>t_{n}\right]} \exp \left(-\log ^{2} n\right) .
\end{aligned}
$$

By Lemma 3.4 and (3.41), equation (3.39) reduces to

$$
\begin{aligned}
\frac{1}{\pi^{G_{n}}(B)} & =O\left(t_{n}\right)+\left(P_{\pi_{B}}^{G_{n}}\left[\widetilde{H}_{B}>t_{n}\right]-O\left(e^{-\log ^{2} n}\right)\right)\left(E_{\sigma_{B}}^{S}\left[H_{B}\right]+O\left(e^{-c \log ^{2} n}\right)\right) \\
& =O\left(t_{n}\right)+\sum_{y \in B} \frac{d_{y}^{n}}{d_{B}^{n}} P_{y}^{G_{n}}\left[\widetilde{H}_{B}>t_{n}\right] E_{\sigma_{B}}^{S}\left[H_{B}\right] .
\end{aligned}
$$

Rearranging of (3.42) yields

$$
\begin{aligned}
\frac{1}{E_{\sigma_{B}}^{S}\left[H_{B}\right]} & =\frac{\pi^{G_{n}}(B) O\left(t_{n}\right)}{E_{\sigma_{B}}^{S}\left[H_{B}\right]}+\sum_{y \in B} \frac{d_{y}^{n} \pi^{G_{n}}(B)}{d_{B}^{n}} P_{y}^{G_{n}}\left[\widetilde{H}_{B}>t_{n}\right] \\
& =O\left(\frac{t_{n}}{n^{2}}\right)+\sum_{y \in B} \frac{d_{y}^{n}}{L_{n}} P_{y}^{G_{n}}\left[\widetilde{H}_{B}>t_{n}\right]
\end{aligned}
$$

where in the last equality we used $\pi^{G_{n}}(B)=\sum_{y \in B} d_{y}^{n} / L_{n}=O\left(n^{-1}\right)$ and $E_{\sigma_{B}}^{S}\left[H_{B}\right] \geq$ $E^{S}\left[H_{B}\right] \geq c^{\prime} n$ by (3.7). Claim (3.36) then follows from (3.43).

Proof of Proposition 3.1. Proposition 3.1 follows directly from Lemmas 3.5 and 3.6.

\section{Degree sequence of the vacant set}

The goal of this section is to control the degree sequence $\mathbf{d}^{n, u}$ of the vacant graph $\mathbf{V}^{n, u}$. We proceed in two steps. First, in Theorem 4.4, we approximate $E^{G_{n}}\left[n_{i}\left(\mathbf{d}^{n, u}\right)\right]$, the expected number of vertices with degree $i \in\{0,1, \ldots, \Delta\}$ in $\mathbf{V}^{n, u}$. Second, in Theorem 4.7, we give a corresponding concentration result.

\subsection{Local coupling with unimodular Galton-Watson tree}

Recall from Section 2.4 that $\mathcal{T}$ denotes the unimodular Galton-Watson tree. We write $P_{x}^{\mathcal{T}}$ for the law of the lazy discrete-time simple random walk $Y=\left(Y_{k}\right)_{k \geq 0}$ on $\mathcal{T}$ started from $x \in \mathcal{T}$ (cf. with the non-lazy random walk $\bar{Y}$ of Section 2.4).

The main ingredient of the proof of Theorem 4.4 is a local coupling of the random multigraph $G_{n}$ and the random walk $X$ with the unimodular Galton-Watson tree $\mathcal{T}$ and the random walk $Y$. In its construction we use the following notation. Given the multigraph $G_{n}$, a random walk $X$ on $G_{n}$, and some $x \in[n]$, we define for $t \in \mathbb{N}$,

$$
G^{X, t}=B(x, 1) \cup \bigcup_{0 \leq k \leq t} B\left(X_{k}, 1\right),
$$


and identify it, as usual, with the corresponding induced subgraph of $G_{n}$. Analogously we define

$$
\mathcal{T}^{Y, t}=B^{\mathcal{T}}(\varnothing, 1) \cup \bigcup_{0 \leq k \leq t} B^{\mathcal{T}}\left(Y_{k}, 1\right),
$$

where $B^{\mathcal{T}}(x, r)$ denotes the ball of radius $r$ around $x$ in the tree $\mathcal{T}$. Recall also that $t_{n}=\log ^{3 l+3} n$.

Lemma 4.1. For every $x \in[n]$ there exists a probability space $\left(\Omega_{x}, \mathcal{F}_{x}, \mathcal{Q}_{x}\right)$, where one can construct a random graph $G_{n}$ together with a process $X$ on $G_{n}$ started at $y \in[n]$, and a random tree $\mathcal{T}$ together with a process $Y$ on $\mathcal{T}$ started at $y^{\prime} \in \mathcal{T}$, such that

(a) $G_{n}$ is $\mathbb{P}^{\mathbf{d}^{n}}$ distributed,

(b) $X$ has the law $P_{y}^{G_{n}}$, where $y$ is a uniformly chosen neighbour of $x$ in $G_{n}$,

(c) $\mathcal{T}$ is the unimodular Galton-Watson tree with offspring distribution $p^{*}$, conditioned on $\left\{\operatorname{deg}(\varnothing)=d_{x}^{n}\right\}$,

(d) $Y$ has the law $P_{y^{\prime}}^{\mathcal{T}}$, where $y^{\prime}$ is a uniformly chosen neighbour of $\varnothing$,

and the event

$$
\mathcal{G}^{t_{n}}=\left\{\begin{array}{l}
\text { There exists a graph isomorphism } \phi \text { of } G^{X, t_{n}} \text { and } \mathcal{T}^{Y, t_{n}} \text { such } \\
\text { that } \phi(x)=\varnothing, \phi(y)=y^{\prime} \text {, and } Y_{k}=\phi\left(X_{k}\right) \text { for all } 0 \leq k \leq t_{n}
\end{array}\right\}
$$

satisfies, for some $c \in(0, \infty)$ and large enough $n$,

$$
\mathcal{Q}_{x}\left[\mathcal{G}^{t_{n}}\right] \geq 1-c n^{-\frac{2}{3}-\frac{\varepsilon_{1}}{2}},
$$

where $\varepsilon_{1}$ is as in Assumption 1.1(b).

Proof. We will construct the coupling on $\left(\Omega_{x}, \mathcal{F}_{x}, \mathcal{Q}_{x}\right)$ by sequentially exploring the graph $G_{n}$ along the trajectory of the random walk $\left(X_{k}\right)_{0 \leq k \leq t_{n}}$. The following algorithm, which provides the construction of $G_{n}$ and $X$, and which has four phases, describes this exploration process. In phase 1 , we construct the graph induced by $B(x, 1)$ for a given $x \in[n]$. In phase 2 and 3 we use the random walk until time $t_{n}$ to continue the construction. In phase 4 we complete $G_{n}$ by the usual pairing construction (see Section 2.2). During the run of the algorithm we distinguish between paired and unpaired half-edges. Note that all random variables in the algorithm are defined on $\left(\Omega_{x}, \mathcal{F}_{x}, \mathcal{Q}_{x}\right)$. We also recall that $H^{n}$ denotes the set of half-edges corresponding to $\mathbf{d}^{n}$ (see (2.14)).

Algorithm 4.2. At the start, all half-edges in $H^{n}$ are set unpaired and $G_{n}=([n], \emptyset)$.

1. For all unpaired half-edges $(x, i \mid$ of the given vertex $x$ :

(i) Choose one half-edge $\left(y^{\prime}, i^{\prime} \mid\right.$ from all unpaired half-edges except $(x, i \mid$ uniformly.

(ii) Pair the half-edges $\left(x, i \mid\right.$ and $\left(y^{\prime}, i^{\prime} \mid\right.$, and add the edge $\left(x, y^{\prime}\right)$ into $G_{n}$.

(iii) If all half-edges incident to $x$ are paired, go to phase 2 .

2. (i) If $B(x, 1) \backslash\{x\}$ is empty, set $X_{0}=x$.

(ii) Otherwise, choose one vertex $y$ uniformly in $B(x, 1) \backslash\{x\}$, and set $X_{0}=y$.

3. For $0 \leq k \leq t_{n}$ :

(i) For all unpaired half-edges $\left(X_{k}, i \mid\right.$ of the vertex $X_{k}$, sequentially: Choose one half-edge $\left(y^{\prime}, i^{\prime} \mid\right.$ from all unpaired half-edges except $\left(X_{k}, i \mid\right.$ uniformly, pair the half-edges $\left(X_{k}, i \mid\right.$ and $\left(y^{\prime}, i^{\prime} \mid\right.$, and add the edge $\left(X_{k}, y^{\prime}\right)$ into $G_{n}$. 
(ii) If all half-edges of $X_{k}$ are paired, sample $X_{k+1}$ using the lazy random walk step distribution started from $X_{k}$.

(iii) If $k=t_{n}$, go to step 4 .

4. Complete $G_{n}$ by the usual pairing construction on still non-paired half-edges, and continue the lazy random walk $X_{k}$ for $k>t_{n}+1$ according to its law.

Obviously, $G_{n}$ and $X$ constructed on $\left(\Omega_{x}, \mathcal{F}_{x}, \mathcal{Q}_{x}\right)$ with the help of this algorithm have properties (a)-(b) claimed in the lemma.

We continue with an estimate of the degree distribution for all vertices discovered in phases 1 and 3. Let

$$
p_{n}^{*}(i-1)=\frac{i n_{i}\left(\mathbf{d}^{n}\right)}{\sum_{1 \leq i \leq \Delta} i n_{i}\left(\mathbf{d}^{n}\right)}
$$

be the distribution of the 'degree minus one' of the vertex corresponding to a uniformly chosen half-edge in $H^{n}$. By Assumption 1.1(b),

$$
p_{n}^{*}(i-1)=\frac{i p_{i}+O\left(n^{-\frac{2}{3}-\varepsilon_{1}}\right)}{\sum_{1 \leq i \leq \Delta} i p_{i}+O\left(n^{-\frac{2}{3}-\varepsilon_{1}}\right)}=p_{i-1}^{*}+O\left(n^{-\frac{2}{3}-\varepsilon_{1}}\right),
$$

where $p^{*}$ is given by definition (1.7). Note that $H_{t_{n}}^{n}$, the set of paired half-edges until time $t_{n}$, satisfies

$$
\left|H_{t_{n}}^{n}\right| \leq 2 \Delta+2 t_{n} \Delta,
$$

where the two terms correspond to the number of half-edges paired in Step 1(ii) and Step 3(i) respectively. Thus, for all half-edges $\left(y^{\prime}, i^{\prime} \mid\right.$ chosen in Step 1(i) and Step 3(i), it holds that

$$
\left|\mathcal{Q}_{x}\left[\operatorname{deg}\left(y^{\prime}\right)=i\right]-p_{n}^{*}(i-1)\right| \leq \frac{2 \Delta+2 t_{n} \Delta}{\left|H^{n} \backslash H_{t_{n}}^{n}\right|} \leq \frac{c t_{n}}{n}, \quad 1 \leq i \leq \Delta .
$$

Therefore, by (4.6),

$$
\left|\mathcal{Q}_{x}\left[\operatorname{deg}\left(y^{\prime}\right)=i\right]-p_{i-1}^{*}\right| \leq c n^{-\frac{2}{3}-\varepsilon_{1}} .
$$

We will now construct the tree $\mathcal{T}$ together with the process $Y$ and the (partial) graph isomorphism $\phi$. We first deal with the case when the coupling succeeds, that is $\mathcal{G}^{t_{n}}$ occurs, and deal with the remaining cases later.

At the beginning of the construction, $\mathcal{T}$ is the graph containing only one vertex, the root $\varnothing$, and we define $\phi(x)=\varnothing$. Then, we add the vertices $z_{1}, \ldots, z_{d_{x}^{n}}$ into $\mathcal{T}$ and connect them to $\varnothing$. If $B(x, 1)$ is a tree, we define

$$
\phi(y)=z_{i}, \quad \text { if }(y, j) \text { is paired with }(x, i) \text { for some } j \in\left[d_{y}^{n}\right] .
$$

We then proceed iteratively along the trajectory of $X$ up to time $t^{*}$ given by

$$
t^{*}=\sup \left\{t \leq t_{n}: G^{X, t^{*}} \text { is a tree }\right\} .
$$

At every time $k \leq t^{*}$, such that $X_{k}$ visits a vertex for the first time, we abbreviate $z=\phi\left(X_{k}\right)$ (which is always defined at this step, by construction), and let $Z_{z}$ be a $p^{*}$ distributed random variable. Due to $(4.9), Z_{z}$ can be coupled with $\operatorname{deg}\left(X_{k}\right)-1$, so that

$$
\mathcal{Q}_{x}\left[Z_{z}=\operatorname{deg}\left(X_{k}\right)-1\right] \geq 1-c n^{-\frac{2}{3}-\varepsilon_{1}},
$$

and we thus do so. We then add $Z_{z}$ new vertices into $\mathcal{T}$ and connect all of them to $z$. If $Z_{z}=\operatorname{deg}\left(X_{k}\right)-1$, we extend $\phi$ by a bijection of the neighbours of $X_{k}$ and of $z$, which is possible since the degrees agree. Finally, we set $Y_{k}=\phi\left(X_{k}\right)=z$. 
If $k>t^{*}$, or if the coupling fails, that is there exists a $k \leq t^{*}$, such that $Z_{\phi\left(X_{k}\right)} \neq$ $\operatorname{deg}\left(X_{k}\right)-1$, we continue the construction of $\mathcal{T}$ and the random walk $Y$ independently of the construction of $G_{n}$ and $X$ in Algorithm 4.2. If the coupling succeeds for all $k \leq t^{*}$ and $t^{*}=t_{n}, G_{n}, X, \mathcal{T}, Y$ satisfy the properties (a)-(d) in Lemma 4.1 and $\mathcal{G}^{t_{n}}$ occurs, by construction.

We thus need to estimate the probability of the event $\{$ the coupling succeeds $\} \cap\left\{t^{*}=\right.$ $\left.t_{n}\right\}$. The event $\left\{t^{*}=t_{n}\right\}$ does not occur only if we choose a half-edge of an already discovered vertex in Steps 1(i) or 3(i). This probability is in every step bounded by $2 \Delta t_{n} /\left|H^{n} \backslash H_{t_{n}}^{n}\right|$ and thus

$$
\mathcal{Q}_{x}\left[t^{*} \neq t_{n}\right] \leq \frac{2 \Delta t_{n}^{2}}{n}
$$

In addition, by (4.9),

$$
\begin{aligned}
\mathcal{Q}_{x}\left[\left\{\exists z \in \mathcal{T}^{Y, t^{*}}: Z_{z} \neq \operatorname{deg}\left(\phi^{-1}(z)\right)-1\right\}\right] & \leq \sum_{z \in \mathcal{T}^{Y, t^{*}}} \mathcal{Q}_{x}\left[Z_{z} \neq \operatorname{deg}\left(\phi^{-1}(z)\right)-1\right] \\
& \leq c t_{n} n^{-\frac{2}{3}-\varepsilon_{1}} \leq c^{\prime \prime} n^{-\frac{2}{3}-\frac{\varepsilon_{1}}{2}}
\end{aligned}
$$

These two estimates imply (4.4), which completes the proof.

Later, for a second moment estimate, we need to couple $G_{n}$ and two random walks started at different vertices $x_{1}, x_{2} \in G_{n}$ with two independent unimodular Galton-Watson trees and random walks on them.

Lemma 4.3. For every $\bar{x}=\left(x_{1}, x_{2}\right), x_{1} \neq x_{2} \in[n]$, there exists a probability space $\left(\Omega_{\bar{x}}, \mathcal{F}_{\bar{x}}, \mathcal{Q}_{\bar{x}}\right)$, where one can construct a random graph $G_{n}$, together with two processes $X^{i}$ on $G_{n}$ started at $y_{i} \in[n]$, for $i \in\{1,2\}$, and two independent random trees $\mathcal{T}^{i}$ with roots $\varnothing_{i}$, together with two processes $Y^{i}$ on $\mathcal{T}^{i}$ started at $y_{i}^{\prime} \in[n]$, for $i \in\{1,2\}$, such that,

(a) $G_{n}$ is $\mathbb{P}^{\mathbf{d}^{n}}$ distributed,

(b) for $i \in\{1,2\}, X^{i}$ has the law $P_{y_{i}}^{G_{n}}$, where $y_{i}$ is a uniformly chosen neighbour of $x_{i}$,

(c) for $i \in\{1,2\}, \mathcal{T}^{i}$ is the unimodular Galton-Watson tree with offspring distribution $p^{*}$, conditioned on $\left\{\operatorname{deg}\left(\varnothing_{i}\right)=d_{x_{i}}^{n}\right\}$,

(d) for $i \in\{1,2\}$, $Y^{i}$ has the law $P_{y_{i}^{\prime}}^{\mathcal{T}}$, where $y_{i}^{\prime}$ is a uniformly chosen neighbour of $\varnothing_{i}$, and the events

$$
\mathcal{G}_{i}^{t_{n}}=\left\{\begin{array}{l}
\text { There exists a graph isomorphism } \phi_{i} \text { of } G^{X^{i}, t_{n}} \text { and } \mathcal{T}^{Y^{i}, t_{n}} \text { such } \\
\text { that } \phi_{i}\left(x_{i}\right)=\varnothing_{i}, \phi\left(y_{i}\right)=y_{i}^{\prime}, \text { and } Y_{k}^{i}=\phi\left(X_{k}^{i}\right), \text { for } 0 \leq k \leq t_{n}
\end{array}\right\}
$$

satisfy, for some $c \in(0, \infty)$ and large enough $n$,

$$
\mathcal{Q}_{\bar{x}}\left[\mathcal{G}_{1}^{t_{n}} \cap \mathcal{G}_{2}^{t_{n}}\right] \geq 1-c n^{-\frac{2}{3}-\frac{\varepsilon_{1}}{2}} .
$$

Proof. The proof is similar to the proof of Lemma 4.1, so we only sketch the differences. Note, that for fixed $x_{1} \neq x_{2}$, we can run phases 1-3 of Algorithm 4.2 twice to construct two subgraphs $G_{n}^{X^{1}, t_{n}}$ and $G_{n}^{X^{2}, t_{n}}$.

The number of paired half-edges in these steps is bounded by $4\left(\Delta+t_{n} \Delta\right)$, that is, similarly as in (4.9), for $1 \leq i \leq \Delta$ and $y^{\prime} \in G_{n}^{X^{1}, t_{n}} \cup G_{n}^{X^{2}, t_{n}}$

$$
\left|\mathcal{Q}_{\bar{x}}\left[\operatorname{deg}\left(y^{\prime}\right)=i\right]-p_{i-1}^{*}\right| \leq c n^{-\frac{2}{3}-\varepsilon_{1}} .
$$


If $G_{n}^{X^{1}, t_{n}}, G_{n}^{X^{2}, t_{n}}$ are disjoint trees, then we can construct two independent trees $\mathcal{T}^{1}, \mathcal{T}^{2}$, together with two random walks $Y^{1}, Y^{2}$, similarly to the proof of Lemma 4.1. If $G_{n}^{X^{1}, t_{n}}$, $G_{n}^{X^{2}, t_{n}}$ are not disjoint, we construct $\mathcal{T}^{1}, \mathcal{T}^{2}, Y^{1}, Y^{2}$ independently. Since, by the same arguments as in (4.13),

$$
\mathcal{Q}_{\bar{x}}\left[G_{n}^{X^{1}, t_{n}}, G_{n}^{X^{2}, t_{n}} \text { are disjoint trees }\right] \geq 1-\frac{c t_{n}^{2}}{n},
$$

this completes the proof of the lemma.

\subsection{Expected degree sequence of the vacant graph}

We now use the coupling from Section 4.1 together with Proposition 3.1, in Theorem 4.4 below, to provide a good approximation for the expectation $E^{G_{n}}\left[n_{i}\left(\mathbf{d}^{n, u}\right)\right]$.

For the rooted tree $\mathcal{T}$ and $A \subset\left[d_{\varnothing}\right]$, we denote by $y_{1}, \ldots, y_{d_{\varnothing}}$ the neighbours of the root $\varnothing$ and for $A \subset\left[d_{\varnothing}\right]$ we define, analogously to (3.1), the set

$$
B_{\varnothing, A}^{\mathcal{T}}=\{\varnothing\} \cup\left\{y_{i}: i \in A\right\} \subset B^{\mathcal{T}}(\varnothing, 1) .
$$

We further set

$$
p_{i}^{u}(\mathcal{T})=\sum_{\substack{C \subset\left[d_{\varnothing}\right] \\|C|=i}} \sum_{\substack{A \subset\left[d_{\varnothing}\right] \\ A \supset C}}(-1)^{|A|-|C|} \exp \left(-u \operatorname{cap}_{\mathcal{T}}\left(B_{\varnothing, A}^{\mathcal{T}}\right)\right)
$$

where the capacity is defined in (2.27).

Theorem 4.4. Let $\varepsilon_{1}$ be as in Assumption 1.1(b). Then for $\delta \in\left(0, \varepsilon_{1} / 4\right)$,

$$
\left.\mid E^{G_{n}}\left[n_{i}\left(\mathbf{d}^{n, u}\right)\right]-n \mathbb{E}^{\mathcal{T}}\left[p_{i}^{u}(\mathcal{T})\right]\right] \mid \leq c n^{\frac{2}{3}-\delta} \quad \mathbb{P}^{\mathbf{d}^{n}} \text {-a.a.s. }
$$

Proof. We start by estimating $\mathbb{E}^{\mathbf{d}^{n}}\left[E^{G_{n}}\left[n_{i}\left(\mathbf{d}^{n, u}\right)\right]\right]$ first. Note that

$$
E^{G_{n}}\left[n_{i}\left(\mathbf{d}^{n, u}\right)\right]=\sum_{x \in[n]} P^{G_{n}}\left[d_{x}^{n, u}=i\right] .
$$

Moreover, by (3.2), if $B(x, 1)$ is a tree, then

$$
P^{G_{n}}\left[d_{x}^{n, u}=i\right]=\sum_{\substack{C \subset\left[d_{x}^{n}\right] \\|C|=i}} \sum_{\substack{A \subset\left[d_{x}^{n}\right] \\ A \supset C}}(-1)^{|A|-|C|} P^{G_{n}}\left[H_{B_{x, A}}>2 u m_{p} n\right] .
$$

We thus control the probability on the right-hand side of (4.23) first. By Assumption 1.1(b),

$$
L_{n}=\sum_{x \in[n]} d_{x}^{n}=\sum_{1 \leq i \leq \Delta} i n_{i}\left(\mathbf{d}^{n, u}\right)=\sum_{i=1}^{\Delta} i\left(n p_{i}+O\left(n^{\frac{1}{3}-\varepsilon_{1}}\right)\right)=n m_{p}+O\left(n^{\frac{1}{3}-\varepsilon_{1}}\right) .
$$

By Assumption 1.1(c), $\mathbb{P}^{\mathbf{d}^{n}}\left[G_{n} \in \mathcal{G}_{t y p}^{\mathbf{d}^{n}}\right] \geq 1-n^{-\frac{2}{3}-\varepsilon_{2}}$. Hence, by Proposition 3.1, for $x \in[n]$ and $A \subset\left[d_{x}^{n}\right]$,

$$
\begin{aligned}
& \mathbb{P}^{\mathbf{d}^{n}}\left[\left|P^{G_{n}}\left[H_{B_{x, A}}>2 u m_{p} n\right]-\exp \left(-2 u \sum_{y \in B_{x, A}} P_{y}^{G_{n}}\left[\widetilde{H}_{B_{x, A}}>t_{n}\right]\right)\right| \leq c n^{-\frac{2}{3}-\varepsilon_{1}}\right] \\
& \geq 1-n^{-\frac{2}{3}-\varepsilon_{2}}
\end{aligned}
$$


where $t_{n}=\log ^{3 l+3} n$ as in Proposition 3.1. Thus, using the coupling of Lemma 4.1, (4.23), (4.25), and the fact that $B(x, 1)$ is a tree on $\mathcal{G}^{t_{n}}$, we have that for every $x \in[n]$ and $\varepsilon_{3}=\min \left\{\varepsilon_{2}, \frac{\varepsilon_{1}}{2}\right\}$, with $\mathcal{Q}_{x}$ probability at least $1-c^{\prime} n^{-\frac{2}{3}-\varepsilon_{3}}$,

$$
\begin{aligned}
& \left|P^{G_{n}}\left[d_{x}^{n, u}=i\right]-\sum_{\substack{C \subset\left[d_{\varnothing}\right] \\
|C|=i}} \sum_{\substack{A \subset\left[d_{\varnothing}\right] \\
A \supset C}}(-1)^{|A|-|C|} \exp \left(-2 u \sum_{y \in B_{\varnothing, A}^{\mathcal{T}}} d_{y}^{n} P_{y}^{\mathcal{T}}\left[\widetilde{H}_{B_{\varnothing, A}^{\mathcal{T}}}^{Y}>t_{n}\right]\right)\right| \\
& \leq c n^{-\frac{2}{3}-\varepsilon_{1}} .
\end{aligned}
$$

In order to approximate the second term in $(4.26)$ by $p_{i}^{u}(\mathcal{T})$ we need the following claim, whose proof is postponed to the end of this section. We recall from Section 2.4 that $\bar{P}_{y}^{\mathcal{T}}$ denotes the law of non-lazy random walk $\bar{Y}$ on $\mathcal{T}$.

Lemma 4.5. For every $y \in B_{\varnothing, A}, 2 P_{y}^{\mathcal{T}}\left[\widetilde{H}_{B_{\varnothing, A}^{\mathcal{T}}}^{Y}=\infty\right]=\bar{P}_{y}^{\mathcal{T}}\left[\widetilde{H}_{B_{\varnothing, A}^{\mathcal{T}}}^{\overline{\mathcal{T}}}=\infty\right]$, and for some $c, c^{\prime} \in(0, \infty)$,

$$
\left|P_{y}^{\mathcal{T}}\left[\widetilde{H}_{B_{\varnothing, A}^{\mathcal{T}}}^{Y}>t_{n}\right]-P_{y}^{\mathcal{T}}\left[\widetilde{H}_{B_{\varnothing, A}^{\mathcal{T}}}^{Y}=\infty\right]\right| \leq c \exp \left(-c^{\prime} t_{n}\right) .
$$

Applying this lemma, (4.20), and (2.27) in inequality (4.26) yields

$$
\mathcal{Q}_{x}\left[\left|P^{G_{n}}\left[d_{x}^{n, u}=i\right]-p_{i}^{u}(\mathcal{T})\right| \leq c n^{-\frac{2}{3}-\varepsilon_{1}}\right] \geq 1-c^{\prime} n^{-\frac{2}{3}-\varepsilon_{3}} .
$$

Coming back to (4.20), to finish the approximation of $\mathbb{E}^{\mathbf{d}^{n}}\left[E\left[n_{i}\left(\mathbf{d}^{n, u}\right)\right]\right]$, we need to sum over all $x \in[n]$. For this we denote by $W_{x}$ the event in (4.28), that is

$$
W_{x}=\left\{\left|P^{G_{n}}\left[d_{x}^{n, u}=i\right]-p_{i}^{u}(\mathcal{T})\right| \leq c n^{-\frac{2}{3}-\varepsilon_{1}}\right\} .
$$

By (4.28), for all $x \in[n]$,

$$
\begin{aligned}
\left|\mathbb{E}^{\mathbf{d}^{n}}\left[P^{G_{n}}\left[d_{x}^{n, u}=i\right]\right]-\mathbb{E}^{\mathcal{T}}\left[p_{i}^{u}(\mathcal{T})\right]\right| & =\mathbb{E}_{\mathcal{Q}_{x}}\left[\left|P^{G_{n}}\left[d_{x}^{n, u}=i\right]-p_{i}^{u}(\mathcal{T})\right|\right] \\
& \leq \mathbb{E}_{\mathcal{Q}_{x}}\left[\left|P^{G_{n}}\left[d_{x}^{n, u}=i\right]-p_{i}^{u}(\mathcal{T})\right| \mathbf{1}_{\left\{W_{x}\right\}}\right]+\mathcal{Q}_{x}\left[W_{x}^{c}\right] \\
& \leq c n^{-\frac{2}{3}-\varepsilon_{1}}+c^{\prime} n^{-\frac{2}{3}-\varepsilon_{3}} \leq c n^{-\frac{2}{3}-\varepsilon_{3}},
\end{aligned}
$$

and thus

$$
\mathbb{E}^{\mathbf{d}^{n}}\left[E^{G_{n}}\left[n_{i}\left(\mathbf{d}^{n, u}\right)\right]\right]=\sum_{x \in[n]} \mathbb{E}^{\mathbf{d}^{n}}\left[P^{G_{n}}\left[d_{x}^{n, u}=i\right]\right]=n \mathbb{E}^{\mathcal{T}}\left[p_{i}^{u}(\mathcal{T})\right]+O\left(n^{\frac{1}{3}-\varepsilon_{3}}\right) .
$$

In order to show (4.21), we need to prove the concentration of $E^{G_{n}}\left[n_{i}\left(\mathbf{d}^{n, u}\right)\right]$ around its mean. We first assume that for every two vertices $x_{1} \neq x_{2} \in[n]$

$$
\begin{aligned}
& \mathbb{E}^{\mathbf{d}^{n}}\left[P^{G_{n}}\left[d_{x_{1}}^{n, u}=i\right] P^{G_{n}}\left[d_{x_{2}}^{n, u}=i\right]\right] \\
& =\mathbb{E}^{\mathbf{d}^{n}}\left[P^{G_{n}}\left[d_{x_{1}}^{n, u}=i\right]\right] \mathbb{E}^{\mathbf{d}^{n}}\left[P^{G_{n}}\left[d_{x_{2}}^{n, u}=i\right]\right]+O\left(n^{-\frac{2}{3}-\frac{\varepsilon_{3}}{2}}\right) .
\end{aligned}
$$

This implies that

$$
\begin{aligned}
& \mathbb{E}^{\mathbf{d}^{n}}\left[E^{G_{n}}\left[n_{i}\left(\mathbf{d}^{n, u}\right)\right]^{2}\right] \\
& =\sum_{x \in[n]} \mathbb{E}^{\mathbf{d}^{n}}\left[P^{G_{n}}\left[d_{x}^{n, u}=i\right]^{2}\right]+\sum_{x \neq y} \mathbb{E}^{\mathbf{d}^{n}}\left[P^{G_{n}}\left[d_{x}^{n, u}=i\right] P^{G_{n}}\left[d_{y}^{n, u}=i\right]\right] \\
& =O(n)+\sum_{x \neq y}\left(\mathbb{E}^{\mathbf{d}^{n}}\left[P^{G_{n}}\left[d_{x}^{n, u}=i\right]\right] \mathbb{E}^{\mathbf{d}^{n}}\left[P^{G_{n}}\left[d_{y}^{n, u}=i\right]\right]+O\left(n^{-\frac{2}{3}-\frac{\varepsilon_{3}}{2}}\right)\right) \\
& =\left(\sum_{x \in[n]} \mathbb{E}^{\mathbf{d}^{n}}\left[P^{G_{n}}\left[d_{x}^{n, u}=i\right]\right]\right)^{2}+O\left(n^{\frac{4}{3}-\frac{\varepsilon_{3}}{2}}\right) \\
& =\mathbb{E}^{\mathbf{d}^{n}}\left[E^{G_{n}}\left[n_{i}\left(\mathbf{d}^{n, u}\right)\right]\right]^{2}+O\left(n^{\frac{4}{3}-\frac{\varepsilon_{3}}{2}}\right),
\end{aligned}
$$


for $0<\varepsilon_{3}<\frac{1}{3}$. Therefore, by the second moment method, for $\delta<\varepsilon_{3} / 4$,

$$
\mathbb{P}^{\mathbf{d}^{n}}\left[\left|E\left[n_{i}\left(\mathbf{d}^{n, u}\right)\right]-\mathbb{E}^{\mathbf{d}^{n}}\left[E\left[n_{i}\left(\mathbf{d}^{n, u}\right)\right]\right]\right|>n^{\frac{2}{3}-\delta}\right]=o(1) .
$$

To show (4.32) consider the coupling $\mathcal{Q}_{\bar{x}}$ from Lemma 4.3 for $\bar{x}=\left(x_{1}, x_{2}\right) \subset[n], x_{1} \neq$ $x_{2}$. Let $\mathcal{T}^{1}$ and $\mathcal{T}^{2}$ be two independent unimodular Galton-Watson trees with offspring distribution $p^{*}$ constructed during the coupling, and define $p_{i}^{u}\left(\mathcal{T}^{1}\right), p_{i}^{u}\left(\mathcal{T}^{2}\right)$ similarly to (4.20), and $W_{x_{1}}, W_{x_{2}}$ similarly to (4.29). By Lemma 4.3, for $\varepsilon_{3}$ as above,

$$
\begin{aligned}
& \left|\mathbb{E}_{\mathcal{Q}_{\bar{x}}}\left[P^{G_{n}}\left[d_{x_{1}}^{n, u}=i\right] P^{G_{n}}\left[d_{x_{2}}^{n, u}=i\right]\right]-\mathbb{E}_{\mathcal{Q}_{\bar{x}}}\left[p_{i}^{u}\left(\mathcal{T}^{1}\right) p_{i}^{u}\left(\mathcal{T}^{2}\right)\right]\right| \\
& \leq \mathbb{E}_{\mathcal{Q}_{\bar{x}}}\left[\left|P^{G_{n}}\left[d_{x_{1}}^{n, u}=i\right] P^{G_{n}}\left[d_{x_{2}}^{n, u}=i\right]-p_{i}^{u}\left(\mathcal{T}^{1}\right) p_{i}^{u}\left(\mathcal{T}^{2}\right)\right| \mathbf{1}_{\left\{W_{x_{1}} \cap W_{x_{2}}\right\}}\right] \\
& \quad+\mathcal{Q}_{\bar{x}}\left[\left(W_{x_{1}} \cap W_{x_{2}}\right)^{c}\right] \\
& \leq c^{\prime} n^{-\frac{2}{3}-\varepsilon_{1}}+c n^{-\frac{2}{3}-\frac{\varepsilon_{3}}{2}} \leq c n^{-\frac{2}{3}-\frac{\varepsilon_{3}}{2}} .
\end{aligned}
$$

Using this, the independence of $p_{i}^{u}\left(\mathcal{T}^{1}\right)$ and $p_{i}^{u}\left(\mathcal{T}^{2}\right)$, and (4.30) in the last line, implies

$$
\begin{array}{r}
\mathbb{E}^{\mathbf{d}^{n}}\left[P^{G_{n}}\left[d_{x_{1}}^{n, u}=i\right] P^{G_{n}}\left[d_{x_{2}}^{n, u}=i\right]\right]=\mathbb{E}_{\mathcal{Q}_{\bar{x}}}\left[p_{i}^{u}\left(\mathcal{T}^{1}\right)\right] \mathbb{E}_{\mathcal{Q}_{\bar{x}}}\left[p_{i}^{u}\left(\mathcal{T}^{2}\right)\right]+O\left(n^{-\frac{2}{3}-\frac{\varepsilon_{3}}{2}}\right) \\
=\mathbb{E}^{\mathbf{d}^{n}}\left[P^{G_{n}}\left[d_{x_{1}}^{n, u}=i\right]\right] \mathbb{E}^{\mathbf{d}^{n}}\left[P^{G_{n}}\left[d_{x_{2}}^{n, u}=i\right]\right]+O\left(n^{-\frac{2}{3}-\frac{\varepsilon_{3}}{2}}\right)
\end{array}
$$

and (4.32) is proved. Thus (4.34) holds, and together with (4.31) this finishes the proof of Theorem 4.4.

It remains to show Lemma 4.5 which we used in the last proof.

Proof of Lemma 4.5. The first claim of the lemma is a simple comparison of lazy and non-lazy random walks. For the second claim we note that by the Markov property, for $y \in B_{\varnothing, A}^{\mathcal{T}}$,

$$
\begin{aligned}
& P_{y}^{\mathcal{T}}\left[\widetilde{H}_{B_{\varnothing, A}^{\mathcal{T}}}>t_{n}\right]-P_{y}^{\mathcal{T}}\left[\widetilde{H}_{B_{\varnothing, A}^{\mathcal{T}}}=\infty\right]=P_{y}^{\mathcal{T}}\left[t_{n}<\widetilde{H}_{B_{\varnothing, A}^{\mathcal{T}}}<\infty\right] \\
& \leq P_{y}^{\mathcal{T}}\left[\operatorname{dist}\left(y, Y_{t_{n}}\right) \leq \frac{t_{n}}{12}\right]+\sup _{x: \operatorname{dist}(\varnothing, x)>\frac{t_{n}}{12}} P_{x}^{\mathcal{T}}\left[\widetilde{H}_{\varnothing}<\infty\right] .
\end{aligned}
$$

We denote by $\mathcal{T}^{3}$ the rooted regular tree with $\operatorname{deg}(x)=3$, for every $x \in \mathcal{T}^{3}$ and root $\varnothing^{\prime}$. Consider the lazy random walk $Y^{\prime}$ on $\mathcal{T}^{3}$ started at $x \in \mathcal{T}^{3}$, and its law $P_{x}^{\mathcal{T}^{3}}$. Since $\operatorname{deg}(x) \geq 3$, for all $x \in \mathcal{T}$, we can couple $Y$ on $\mathcal{T}$ with $Y^{\prime}$ on $\mathcal{T}^{3}$, so that, a.s., $\operatorname{dist}\left(\varnothing, Y_{k}\right) \geq \operatorname{dist}\left(\varnothing^{\prime}, Y_{k}^{\prime}\right)$. Hence,

$$
\begin{aligned}
P_{y}^{\mathcal{T}}\left[\operatorname{dist}\left(\varnothing, Y_{t_{n}}\right) \leq \frac{t_{n}}{12}\right] & \leq P_{y}^{\mathcal{T}^{3}}\left[\operatorname{dist}\left(\varnothing^{\prime}, Y_{t_{n}}^{\prime}\right) \leq \frac{t_{n}}{12}\right], \\
\sup _{x: \operatorname{dist}(\varnothing, x)>\frac{t_{n}}{12}} P_{x}^{\mathcal{T}}\left[\widetilde{H}_{\varnothing}<\infty\right] & \leq \sup _{x: \operatorname{dist}(\varnothing, x)>\frac{t_{n}}{12}} P_{x}^{\mathcal{T}^{3}}\left[\widetilde{H}_{\varnothing}<\infty\right] .
\end{aligned}
$$

Note that $\operatorname{dist}\left(\varnothing, Y_{t_{n}}^{\prime}\right)$ under $P_{y}^{\mathcal{T}^{3}}$ is a lazy random walk on $\mathbb{N}$ with the expected drift given by $\frac{1}{6}$. Standard large deviation estimates for this walk together with (4.38) and (4.39) then imply the lemma.

\subsection{Concentration of the degree sequence}

In this section we show that $n_{i}\left(\mathbf{d}^{n, u}\right)$ concentrates around its mean. 
Theorem 4.6. For every $u \geq 0$ and $\varepsilon>0$ there exist $c, c^{\prime} \in(0, \infty)$ such that for every $n \geq 2, i \in\{0, \ldots, \Delta\}, \varepsilon \in(0,1 / 2)$ and $G_{n} \in \mathcal{G}_{\text {typ }}^{\mathbf{d}^{n}}$,

$$
P^{G_{n}}\left[\left|n_{i}\left(\mathbf{d}^{n, u}\right)-E^{G_{n}}\left[n_{i}\left(\mathbf{d}^{n, u}\right)\right]\right| \geq n^{\frac{1}{2}+\varepsilon}\right] \leq c^{\prime} \exp \left(-c n^{\varepsilon / 2}\right) .
$$

The proof of of this theorem uses similar ideas as in [ČTW11] and is based on the following well known concentration inequality.

Lemma 4.7 (Theorem 3.7 of [McD98]). Let $W=\left(W_{1}, \ldots, W_{M}\right)$ be a family of random variables taking values in some measurable space $(S, \mathcal{S}), f: S^{M} \rightarrow \mathbb{R}$ be a bounded function, and let $\mu=E[f(W)]$ be the mean of $f(W)$. Define

$$
\begin{aligned}
& r_{k}\left(w_{1}, \ldots, w_{k-1}\right) \\
& =\sup _{y, y^{\prime} \in S}\left|E\left[f(W) \mid W_{k}=y, W_{i}=w_{i} \forall i<k\right]-E\left[f(W) \mid W_{k}=y^{\prime}, W_{i}=w_{i} \forall i<k\right]\right| .
\end{aligned}
$$

Then, for any $t \geq 0$ and $R^{2}=\sup _{w_{1}, \ldots, w_{M-1}} \sum_{k=1}^{M} r_{k}^{2}\left(w_{1}, \ldots, w_{k-1}\right)$,

$$
P[|f(W)-\mu| \geq t] \leq 2 \exp \left(-\frac{t^{2}}{R^{2}}\right) .
$$

To apply this inequality we replace the random walk $X$ on $G_{n}$ by another process obtained by concatenating random walk bridges of length $L=\left\lfloor n^{\delta}\right\rfloor$. Let $G_{n} \in \mathcal{G}_{\text {typ }}^{\mathbf{d}^{n}}$. For $x, z \in[n]$, denote by $P_{x}^{G_{n}, L}$ the law of the random walk $\left(X_{k}\right)_{0 \leq k \leq L}$ on $G_{n}$ started at $x$, and by $P_{x, z}^{G_{n}, L}=P_{x}^{G_{n}, L}\left[\cdot \mid X_{L}=z\right]$ the law of the corresponding random walk bridge. Let $\left(Z^{i}\right)_{i \geq 0}$ be a sequence of $\pi^{G_{n}}$-distributed random variables defined on some auxiliary probability space $(\tilde{\Omega}, \tilde{\mathcal{A}}, \tilde{P})$. Given $\left(Z^{i}\right)_{i \geq 0}$, let $\left(\bar{X}^{i}\right)_{i \geq 1}$ be conditionally independent elements of $[n]^{L+1}$, defined on the same auxiliary probability space, such that each $\left(\bar{X}_{k}^{i}\right)_{0 \leq k \leq L}$ is distributed according to the random walk bridge measure $P_{Z^{i-1}, Z^{i}}^{G_{n}, L}$. We define the concatenation of the $\bar{X}^{i}$ as

$$
\mathcal{X}_{k}=\bar{X}_{k-(i-1) L}^{i}, \quad \text { for }(i-1) L \leq k<i L .
$$

For $\kappa \geq 0$, let $\mathcal{P}^{G_{n}, \kappa}$ be the law of $\left(\mathcal{X}_{0}, \ldots, \mathcal{X}_{\lfloor\kappa n\rfloor}\right)$ on $[n]^{\lfloor\kappa n\rfloor+1}$, and write $P^{G_{n}, \kappa}$ for the law of the random walk $\left(X_{0}, \ldots, X_{\lfloor\kappa n\rfloor}\right)$. The next lemma shows that $\mathcal{P}^{G_{n}, \kappa}$ approximates well $P^{G_{n}, \kappa}$.

Lemma 4.8. For every $\kappa \geq 0$ and $G_{n} \in \mathcal{G}_{\text {typ }}^{\mathbf{d}^{n}}$, with $L=\left\lfloor n^{\delta}\right\rfloor, \delta>0$, the measures $P^{G_{n}, \kappa}$ and $\mathcal{P}^{G_{n}, \kappa}$ are equivalent, and for some $c, c^{\prime}>0$ independent of $n$

$$
\left|\frac{P^{G_{n}, \kappa}[A]}{\mathcal{P}^{G_{n}, \kappa}[A]}-1\right| \leq c \exp \left(-c^{\prime} n^{\frac{\delta}{2}}\right), \quad \text { for every } A \subseteq[n]^{\lfloor\kappa n\rfloor+1} .
$$

Proof. Let $\kappa^{\prime}$ be the smallest number, such that $\kappa^{\prime} \geq \kappa$ and $m L=\kappa^{\prime} n$ for some $m \in \mathbb{N}$. Since $P^{G_{n}, \kappa}$ and $\mathcal{P}^{G_{n}, \kappa}$ are restrictions of $P^{G_{n}, \kappa^{\prime}}$ and $\mathcal{P}^{G_{n}, \kappa^{\prime}}$, it is enough to prove (4.42) for $P^{G_{n}, \kappa^{\prime}}$ and $\mathcal{P}^{G_{n}, \kappa^{\prime}}$. Let $A$ be an arbitrary subset of $[n]^{\left\lfloor\kappa^{\prime} n\right\rfloor+1}$. Then, by the Markov property,

$$
\begin{aligned}
P^{G_{n}, \kappa^{\prime}}[A] & \\
& =\sum_{x_{0}, \ldots, x_{m} \in[n]} P^{G_{n}, \kappa^{\prime}}\left[A \mid X_{k L}=x_{k}, 0 \leq k \leq m\right] P^{G_{n}, \kappa^{\prime}}\left[X_{k L}=x_{k}, 0 \leq k \leq m\right] \\
& =\sum_{x_{0}, \ldots, x_{m} \in[n]} P^{G_{n}, \kappa^{\prime}}\left[A \mid X_{k L}=x_{k}, 0 \leq k \leq m\right] \pi^{G_{n}}\left(x_{0}\right) \prod_{i=1}^{m} P_{x_{i-1}}^{G_{n}}\left[X_{L}=x_{i}\right] .
\end{aligned}
$$


On the other hand, the construction of $\mathcal{P}^{G_{n}, \kappa}$ implies that

$$
\begin{aligned}
\mathcal{P}^{G_{n}, \kappa^{\prime}}[A] & \\
& =\sum_{x_{0}, \ldots, x_{m} \in[n]} \mathcal{P}^{G_{n}, \kappa^{\prime}}\left[A \mid X_{k L}=x_{k}, 0 \leq k \leq m\right] \mathcal{P}^{G_{n}, \kappa^{\prime}}\left[X_{k L}=x_{k}, 0 \leq k \leq m\right] \\
& =\sum_{x_{0}, \ldots, x_{m} \in[n]} P^{G_{n}, \kappa^{\prime}}\left[A \mid X_{k L}=x_{k}, 0 \leq k \leq m\right] \prod_{i=0}^{m} \pi^{G_{n}}\left(x_{i}\right) .
\end{aligned}
$$

Moreover, by (2.10), since $G_{n} \in \mathcal{G}_{\text {typ }}^{\mathbf{d}^{n}}$ and $L=\left\lfloor n^{\delta}\right\rfloor$, for every $x, y \in[n]$, for $n$ large enough,

$$
\left|\frac{P_{x}^{G_{n}}\left[X_{L}=y\right]}{\pi^{G_{n}}(y)}-1\right| \leq c n e^{-\lambda_{G_{n}} L} \leq c n e^{-c L \log ^{-l} n} \leq c n e^{-c n^{\delta / 2}} .
$$

Combining (4.43)-(4.45) then yields

$$
\left(1-c^{\prime} n \exp \left(-c n^{\frac{\delta}{2}}\right)\right)^{m} \leq \frac{P^{G_{n}, \kappa^{\prime}}[A]}{\mathcal{P}^{G_{n}, \kappa^{\prime}}[A]} \leq\left(1+c^{\prime} n \exp \left(-c n^{\frac{\delta}{2}}\right)\right)^{m},
$$

and (4.42) follows by possibly changing the constants.

Proof of Theorem 4.6. Set $\kappa=2 u m_{p}, m=\lceil\kappa n / L\rceil$, and $\kappa^{\prime}=m L / n$. Denoting by $\mathcal{E}^{G_{n}, \kappa^{\prime}}$ the expectation corresponding to $\mathcal{P}^{G_{n}, \kappa^{\prime}}$, for $G_{n} \in \mathcal{G}_{\text {typ }}^{\mathbf{d}^{n}}$, by Lemma 4.8 with $\varepsilon=\delta$,

$$
\left|E^{G_{n}}\left[n_{i}\left(\mathbf{d}^{n, u}\right)\right]-\mathcal{E}^{G_{n}, \kappa^{\prime}}\left[n_{i}\left(\mathbf{d}^{n, u}\right)\right]\right| \leq c n \exp \left(-c^{\prime} n^{\frac{\varepsilon}{2}}\right) .
$$

Therefore, applying once more Lemma 4.8, for $n$ large enough,

$$
\begin{aligned}
& P^{G_{n}}\left[\left|n_{i}\left(\mathbf{d}^{n, u}\right)-E^{G_{n}}\left[n_{i}\left(\mathbf{d}^{n, u}\right)\right]\right| \geq n^{\frac{1}{2}+\varepsilon}\right] \\
& \leq \mathcal{P}^{G_{n}, \kappa^{\prime}}\left[\left|n_{i}\left(\mathbf{d}^{n, u}\right)-\mathcal{E}^{G_{n}, \kappa^{\prime}}\left[n_{i}\left(\mathbf{d}^{n, u}\right)\right]\right| \geq 2 n^{\frac{1}{2}+\varepsilon}\right]+c \exp \left(-c^{\prime} n^{\frac{\varepsilon}{2}}\right) .
\end{aligned}
$$

We now apply Lemma 4.7 with $M=m, S=[n]^{L+1}, W_{k}=\bar{X}^{k}$ and $f(W)=n_{i}\left(\mathbf{d}^{n, u}\right)$. Denoting by $F_{i}^{j}=F_{i}^{j}\left(w_{i}, \ldots, w_{j}\right)$ the event $\left\{\bar{X}^{k}=w_{k} \forall i \leq k \leq j\right\}$, we claim that for all $k=1, \ldots, m$ and $w_{1}, \ldots, w_{k-1} \in S$

$$
\begin{aligned}
& r_{k}\left(w_{1}, \ldots, w_{k-1}\right) \\
& =\sup _{w, w^{\prime} \in S}\left|\mathcal{E}^{G_{n}, \kappa^{\prime}}\left[n_{i}\left(\mathbf{d}^{n, u}\right) \mid \bar{X}^{k}=w, F_{1}^{k-1}\right]-\mathcal{E}^{G_{n}, \kappa^{\prime}}\left[n_{i}\left(\mathbf{d}^{n, u}\right) \mid \bar{X}^{k}=w^{\prime}, F_{1}^{k-1}\right]\right| \\
& \leq(\Delta+1) L .
\end{aligned}
$$

Indeed, when conditioning additionally on $F_{k+1}^{m}=\left\{\bar{X}^{k+1}=w_{k+1}, \ldots, \bar{X}^{m}=w_{m}\right\}$, since $\bar{X}^{k}$ contains at most $L$ different vertices and thus it can change the degree in the vacant graph of at most $(\Delta+1) L$ vertices,

$$
\begin{aligned}
& \left|\mathcal{E}^{G_{n}, \kappa^{\prime}}\left[n_{i}\left(\mathbf{d}^{n, u}\right) \mid \bar{X}^{k}=w, F_{1}^{k-1} \cap F_{k+1}^{m}\right]-\mathcal{E}^{G_{n}, \kappa^{\prime}}\left[n_{i}\left(\mathbf{d}^{u^{\prime}, n}\right) \mid \bar{X}^{k}=w^{\prime}, F_{1}^{k-1} \cap F_{k+1}^{m}\right]\right| \\
& \leq(\Delta+1) L .
\end{aligned}
$$

Integrating over $\bar{X}^{k+1}, \ldots, \bar{X}^{m}$ then implies (4.49).

Using (4.49), we can apply Lemma 4.7 with $R^{2} \leq m(\Delta+1)^{2} L^{2} \leq c \frac{u n}{L} L^{2}=c n^{1+\varepsilon}$ to obtain

$$
\mathcal{P}^{G_{n}, \kappa^{\prime}}\left[\left|n_{i}\left(\mathbf{d}^{u, n}\right)-\mathcal{E}^{G_{n}, \kappa^{\prime}}\left[n_{i}\left(\mathbf{d}^{u, n}\right)\right]\right| \geq c n^{\frac{1}{2}+\varepsilon}\right] \leq 2 \exp \left(-\frac{c n^{1+2 \varepsilon}}{n^{1+\varepsilon}}\right)=c e^{-c^{\prime} n^{\varepsilon}} .
$$

Combining (4.48) and (4.51) proves Theorem 4.6. 


\section{$5 \quad$ Proofs of Theorem 1.2 and 1.3}

In this section we show the main results of this paper, Theorems 1.2 and 1.3. As said in the introduction, these theorems essentially follow from Proposition 2.1 and Theorem 2.2, if we estimate the parameter $Q\left(\mathbf{d}^{n, u}\right)$ sufficiently precisely; here $\mathbf{d}^{n, u}$ is the degree sequence of the vacant graph and $Q$ is as in (2.20).

Observe that

$$
Q\left(\mathbf{d}^{n, u}\right)=\frac{\sum_{x=1}^{n}\left(d_{x}^{n, u}\right)^{2}}{\sum_{x=1}^{n} d_{x}^{n, u}}=\frac{\sum_{i=0}^{\Delta} i^{2} n_{i}\left(\mathbf{d}^{n, u}\right)}{\sum_{i=0}^{\Delta} i n_{i}\left(\mathbf{d}^{n, u}\right)} .
$$

Moreover, by Theorems 4.4, 4.6, there exist $c, \delta \in(0, \infty)$ such that for $u>0$ and $i \in\{0,1, \ldots, \Delta\}$,

$$
\left|n_{i}\left(\mathbf{d}^{n, u}\right)-n \mathbb{E}^{\mathcal{T}}\left[p_{i}^{u}(\mathcal{T})\right]\right| \leq c n^{\frac{2}{3}-\delta}, \quad \mathbf{P}_{n^{-a} \text { a.a.s. }}
$$

where $p_{i}^{u}(\mathcal{T})$ is as in $(4.20)$.

To compute the right-hand side of (5.1), we need a last technical lemma which will be shown at the end of this section. For its statement recall the notation of Section 2.4.

Lemma 5.1. Let $u>0$ and $\phi(u)$ be as in (2.29). Then

$$
\mathbb{E}^{\mathcal{T}}\left[p_{i}^{u}(\mathcal{T})\right]=\mathbb{E}\left[\phi(u)^{D}\left(\begin{array}{c}
D \\
i
\end{array}\right) \mathbb{E}\left[\phi(u)^{D^{*}-1}\right]^{i}\left(1-\mathbb{E}\left[\phi(u)^{D^{*}-1}\right]\right)^{D-i}\right],
$$

where $D$ and $D^{*}$ are random variables distributed according to $p$ and $p^{*}$, respectively. In particular,

$$
\frac{\sum_{i=0}^{\Delta} i^{2} \mathbb{E}^{\mathcal{T}}\left[p_{i}^{u}(\mathcal{T})\right]}{\sum_{i=0}^{\Delta} i \mathbb{E}^{\mathcal{T}}\left[p_{i}^{u}(\mathcal{T})\right]}=m_{p}^{-1} \sum_{i=3}^{\Delta} p_{i} i(i-1) \phi(u)^{i-2}+1
$$

From this lemma, (5.1), and (5.2), it follows that for $u>0$,

$$
\left|Q\left(\mathbf{d}^{n, u}\right)-\left(m_{p}^{-1} \sum_{i=3}^{\Delta} p_{i} i(i-1) \phi(u)^{i-2}+1\right)\right| \leq c n^{-1 / 3-\delta}, \quad \mathbf{P}_{n^{-}} \text {a.a.s. }
$$

We can now finally prove our main results.

Proof of Theorem 1.2. We start by proving parts (a) and (b) of the theorem which follow essentially directly from parts (a) and (b) of Theorem 2.2. By Proposition 2.1, the vacant graph $\mathbf{V}^{n, u}$ is distributed as the random (multi)graph with the random degree sequence $\mathbf{d}^{n, u}$. Moreover, by (5.2), the assumptions of Theorem 2.2 are $\mathbf{P}_{n}$-a.s.s. satisfied for the degree sequence $\mathbf{d}^{n, u}$ with $p_{i}$ of $(2.21)$ being $\mathbb{E}^{\mathcal{T}}\left(p_{i}^{u}(\mathcal{T})\right)$. By (5.5), there is a non-random $Q^{u}$ such that $Q^{u}=\lim _{n \rightarrow \infty} Q\left(\mathbf{d}^{n, u}\right), \mathbf{P}_{n}$-a.a.s. In addition, $Q^{u^{*}}=2$ iff $u^{*}$ is the solution of equation (2.31) and $Q^{u}>2$ iff $u<u^{*}$. Thus parts (a) and (b) of Theorem 1.2 follow from (a) and (b) of Theorem 2.2.

We now prove the part (c). Let $u^{*}$ be the solution of equation (2.31) and recall that $u_{n} \rightarrow u^{*}$ satisfies

$$
n^{1 / 3}\left|u^{*}-u_{n}\right| \leq \eta<\infty .
$$

Then, by expanding the exponential $\exp \left(-u \bar{P}_{\mathscr{\chi}^{\prime}}^{\tilde{\mathcal{T}}}\left[H_{\tilde{\varnothing}}^{\bar{Y}}=\infty\right]\right)$ appearing in the definition (2.29) of $\phi$ around $u^{*}$, and by using (2.31), we find that the first summand on the right-hand side of (5.4) satisfies

$$
\begin{aligned}
m_{p}^{-1} \sum_{i=3}^{\Delta} p_{i} i(i-1) \phi\left(u_{n}\right)^{i-2} & =m_{p}^{-1} \sum_{i=3}^{\Delta} p_{i} i(i-1) \mathbb{E}^{\tilde{\mathcal{T}}}\left[\exp \left(-u_{n} \bar{P}_{\varnothing^{\prime}}^{\tilde{\mathcal{T}}}\left[H_{\tilde{\varnothing}}^{\bar{Y}}=\infty\right]\right)\right]^{i-2} \\
& =1-c_{p}\left(u^{*}-u_{n}\right)+O\left(\left(u^{*}-u_{n}\right)^{2}\right)
\end{aligned}
$$


for some constant $c_{p}>0$ depending only on the distribution $p$. With (5.5) this yields

$$
\left|Q\left(\mathbf{d}^{u_{n}, n}\right)-2\right| \leq 2 c_{p} \eta n^{-\frac{1}{3}}, \quad \mathbf{P}_{n}-\text { a.a.s. }
$$

Applying Theorem 2.2(c) together with Proposition 2.1 implies the claim (c) of Theorem 1.2.

The claim (d) follows directly from the facts that the critical point of random interlacements on $\mathcal{T}$ agrees with the critical point of random interlacements on $\mathcal{T}^{\prime}$ (see the proof of Proposition 2.3), and both of them, as well as the $u^{*}$ of the present theorem, are given as the unique solution of (2.31).

Remark 5.2. The constant $\rho$ of Theorem 1.2(a) can be identified semi-explicitely using Theorem 2.2 and (5.3). Namely, letting $g(x)=\sum_{i=0}^{\Delta} \mathbb{E}^{\mathcal{T}}\left[p_{i}^{u}(\mathcal{T})\right] x^{i}$ to be the generating function of the limiting distribution of the degree sequence of the vacant graph (see (5.2)), then $\rho=1-g(\xi)$ with $\xi$ being the unique solution in $(0,1)$ of the equation $g^{\prime}(\xi)=\xi \sum_{i=0}^{\Delta} i \mathbb{E}^{\mathcal{T}}\left[p_{i}^{u}(\mathcal{T})\right]$.

Proof of Theorem 1.3. The proof follows essentially the same steps as the proof of Theorem 1.2(c), with the stronger assumption

$$
u_{n}=u^{*}+\eta n^{-\frac{1}{3}}+o\left(n^{-1 / 3}\right) .
$$

Using the same steps as for (5.8), this assumption implies that

$$
Q\left(\mathbf{d}^{u_{n}, n}\right)=2+c_{p} \eta n^{-1 / 3}+o\left(n^{-1 / 3}\right)
$$

With this at hand, we can apply part (d) of Theorem 2.2 together with Proposition 2.1 to complete the proof of Theorem 1.3.

Proof of Lemma 5.1. We write $\widetilde{H}_{A}$ for $\widetilde{H}_{A}^{\bar{Y}}$ in this proof and use notation from (4.19). Note that, for $y \in B_{\varnothing, A}^{\mathcal{T}} \backslash\{\varnothing\}$, there exists a $j \in A$, such that $y_{j}=y$. Then, by the Markov property,

$$
d_{\varnothing} \bar{P}_{\varnothing}^{\mathcal{T}}\left[\widetilde{H}_{B_{\varnothing, A}^{\mathcal{T}}}=\infty\right]=\sum_{j \in\left[d_{\varnothing}\right] \backslash A} \bar{P}_{y_{j}}^{\mathcal{T}}\left[\widetilde{H}_{\varnothing}=\infty\right] .
$$

Using this in the second step of the next computation we obtain

$$
\begin{aligned}
\operatorname{cap}_{\mathcal{T}}\left(B_{\varnothing, A}^{\mathcal{T}}\right) & =\sum_{y \in B_{\varnothing, A}^{\mathcal{T}}} d_{y} \bar{P}_{y}^{\mathcal{T}}\left[\widetilde{H}_{B_{\varnothing, A}^{\mathcal{T}}}=\infty\right] \\
& =\sum_{j \in A} d_{y_{j}} \bar{P}_{y_{j}}^{\mathcal{T}}\left[\widetilde{H}_{\left\{\varnothing, y_{j}\right\}}=\infty\right]+d_{\varnothing} \bar{P}_{\varnothing}^{\mathcal{T}}\left[\widetilde{H}_{B_{\varnothing, A}^{\mathcal{T}}}=\infty\right] \\
& =\sum_{j \in A} d_{y_{j}} \bar{P}_{y_{j}}^{\mathcal{T}}\left[\widetilde{H}_{\left\{\varnothing, y_{j}\right\}}=\infty\right]+\sum_{j \in\left[d_{\varnothing}\right] \backslash A} \bar{P}_{y_{j}}^{\mathcal{T}}\left[\widetilde{H}_{\varnothing}=\infty\right] .
\end{aligned}
$$

The probability $\bar{P}_{y_{i}}^{\mathcal{T}}\left[\widetilde{H}_{\varnothing}=\infty\right]$ only depends on the subtree $\mathcal{T}_{y_{i}} \subset \mathcal{T}$, containing the vertex $y_{i} \sim \varnothing$ and all its descendants. Thus $\bar{P}_{y_{i}}^{\mathcal{T}}\left[\widetilde{H}_{\varnothing}=\infty\right], i=1, \ldots,\left[d_{\varnothing}\right]$, are conditionally i.i.d. given $d_{\varnothing}$. The same holds for $\bar{P}_{y_{i}}^{\mathcal{T}}\left[\widetilde{H}_{\left\{\varnothing, y_{i}\right\}}=\infty\right]$. Thus, inserting (5.12) into (4.20) 
gives

$$
\begin{aligned}
\mathbb{E}^{\mathcal{T}}\left[p_{i}^{u}(\mathcal{T})\right] & =\mathbb{E}^{\mathcal{T}}\left[\sum_{\substack{C \subset\left[d_{\varnothing}\right] \\
|C|=i}} \sum_{\substack{A \subset\left[d_{\varnothing}\right] \\
A \supset C}}(-1)^{|A|-|C|} \exp \left(-u \operatorname{cap}_{\mathcal{T}}\left(B_{\varnothing, A}^{\mathcal{T}}\right)\right)\right] \\
= & \mathbb{E}^{\mathcal{T}}\left[\sum_{\substack{C \subset\left[d_{\varnothing}\right] \\
|C|=i}} \sum_{\substack{A \subset\left[d_{\varnothing}\right] \\
A \supset C}}(-1)^{|A|-|C|} \prod_{j \in A} \mathbb{E}^{\mathcal{T}}\left[\exp \left(-u d_{y_{j}} \bar{P}_{y_{j}}^{\mathcal{T}}\left[\widetilde{H}_{\left\{\varnothing, y_{j}\right\}}=\infty\right]\right) \mid d_{\varnothing}\right]\right. \\
& \left.\times \prod_{j \in\left[d_{\varnothing}\right] \backslash A} \mathbb{E}^{\mathcal{T}}\left[\exp \left(-u \bar{P}_{y_{j}}^{\mathcal{T}}\left[\widetilde{H}_{\varnothing}=\infty\right]\right) \mid d_{\varnothing}\right]\right] .
\end{aligned}
$$

Since $\mathcal{T}$ is a unimodular Galton-Watson tree, for every $y \sim \varnothing, z \sim y, z \neq \varnothing$, the laws of $\mathcal{T}_{y} \cup\{\varnothing\}$ conditioned on $d_{\varnothing}$ and of $\mathcal{T}_{z} \cup\{y\}$ conditioned on $d_{\varnothing}$ and $d_{y}$ agree with the law of $\tilde{\mathcal{T}}$ introduced before the definition (2.29) of $\phi(u)$. Therefore, for every such $y$ and $z$,

$$
\mathbb{E}^{\mathcal{T}}\left[\exp \left(-u \bar{P}_{y}^{\mathcal{T}}\left[\widetilde{H}_{\varnothing}=\infty\right]\right) \mid d_{\varnothing}\right]=\mathbb{E}^{\mathcal{T}}\left[\exp \left(-u \bar{P}_{z}^{\mathcal{T}}\left[\widetilde{H}_{y}=\infty\right]\right) \mid d_{y}, d_{\varnothing}\right]=\phi(u) .
$$

Similarly, by the Markov property, the usual independence arguments, and (5.14), for $y \sim \varnothing$

$$
\begin{aligned}
\mathbb{E}^{\mathcal{T}} & {\left[\exp \left(-u d_{y} \bar{P}_{y}^{\mathcal{T}}\left[\widetilde{H}_{\{\varnothing, y\}}=\infty\right]\right) \mid d_{\varnothing}\right]=\mathbb{E}^{\mathcal{T}}\left[\exp \left(-u \sum_{z \sim y, z \neq \varnothing} \bar{P}_{z}^{\mathcal{T}}\left[\widetilde{H}_{y}=\infty\right]\right) \mid d_{\varnothing}\right] } \\
= & \mathbb{E}^{\mathcal{T}}\left[\phi(y)^{d_{y}-1} \mid d_{\varnothing}\right]=\mathbb{E}^{\mathcal{T}}\left[\phi(y)^{d_{y}-1}\right],
\end{aligned}
$$

since the degree of any $y \sim \varnothing$ is independent of $d_{\varnothing}$. Using (5.14) and (5.15), and noting $\mathbb{E}^{\mathcal{T}}\left[\phi(u)^{d_{y_{i}}-1}\right]=\phi(u) \mathbb{E}^{\mathcal{T}}\left[\phi(u)^{d_{y_{i}}-2}\right]$ since $d_{y_{i}} \geq 2$, (5.13) simplifies to

$$
\begin{aligned}
\mathbb{E}^{\mathcal{T}}\left[p_{i}^{u}(\mathcal{T})\right] & =\mathbb{E}^{\mathcal{T}}\left[\sum_{\substack{C \subset\left[d_{\varnothing}\right] \\
|C|=i}} \sum_{\substack{A \subset\left[d_{\varnothing}\right] \\
A \supset C}}(-1)^{|A|-|C|} \prod_{j \in A} \mathbb{E}^{\mathcal{T}}\left[\phi(u)^{d_{y_{j}}-1}\right] \prod_{j \in\left[d_{\varnothing}\right] \backslash A} \phi(u)\right] \\
& =\mathbb{E}^{\mathcal{T}}\left[\prod_{j \in\left[d_{\varnothing}\right]} \phi(u) \sum_{\substack{C \subset\left[d_{\varnothing \varnothing}\right] \\
|C|=i}} \sum_{\substack{A \subset\left[d_{\varnothing}\right] \\
A \supset C}}(-1)^{|A|-|C|} \prod_{j \in A} \mathbb{E}^{\mathcal{T}}\left[\phi(u)^{d_{y_{j}}-2}\right]\right] \\
& =\mathbb{E}^{\mathcal{T}}\left[\phi(u)^{d_{\varnothing}}\left(\begin{array}{c}
d_{\varnothing} \\
i
\end{array}\right) \mathbb{E}^{\mathcal{T}}\left[\phi(u)^{d_{y}-2}\right]^{i}\left(1-\mathbb{E}^{\mathcal{T}}\left[\phi(u)^{d_{y}-2}\right]\right)^{d_{\varnothing}-i}\right],
\end{aligned}
$$

where in the last line $y$ is an arbitrary neighbour of $\varnothing$. This proves (5.3), since $d_{\varnothing}$ is $p$ distributed, and for $y \sim \varnothing, d_{y}=1+$ 'number of offsprings of $y$ ' has the same distribution as $1+D^{\star}$, with $D^{\star}$ being $p^{*}$-distributed.

Using (5.3), writing the distribution of $D$ explicitely, recalling that $p_{1}=p_{2}=0$ by assumption, the numerator of (5.4) can be written as

$$
\begin{aligned}
\sum_{i=0}^{\Delta} i^{2} \mathbb{E}^{\mathcal{T}}\left[p_{i}^{u}(\mathcal{T})\right] & =\sum_{k=3}^{\Delta} p_{k} \phi(u)^{k} \sum_{i=0}^{k} i^{2}\left(\begin{array}{c}
k \\
i
\end{array}\right) \mathbb{E}\left[\phi(u)^{D^{*}-1}\right]^{i}\left(1-\mathbb{E}\left[\phi(u)^{D^{*}-1}\right]\right)^{k-i} \\
& =\sum_{k=3}^{\Delta} p_{k} \phi(u)^{k} k \mathbb{E}\left[\phi(u)^{D^{*}-1}\right]\left((k-1) \mathbb{E}\left[\phi(u)^{D^{*}-1}\right]+1\right)
\end{aligned}
$$

where for the second equality we used the fact that the inner sum is the second moment of the binomial distribution with parameters $k$ and $\mathbb{E}\left[\phi(u)^{D^{*}-1}\right]$. Similarly, the 
denominator of (5.4) can be written as

$$
\sum_{i=0}^{\Delta} i \mathbb{E}^{\mathcal{T}}\left[p_{i}^{u}(\mathcal{T})\right]=\sum_{k=3}^{\Delta} p_{k} \phi(u)^{k} k \mathbb{E}\left[\phi(u)^{D^{*}-1}\right] .
$$

Finally, using the definition (1.7) of the offspring distribution $p^{*}$, we obtain

$$
\mathbb{E}^{\mathcal{T}}\left[\phi(u)^{D^{*}-1}\right]=\sum_{k=2}^{\Delta} p_{k}^{*} \phi(u)^{k-1}=\sum_{k=3}^{\Delta} k p_{k} m_{p}^{-1} \phi(u)^{k-2} .
$$

Combining (5.17), (5.18) and (5.19) then yields

$$
\begin{aligned}
\frac{\sum_{i=0}^{\Delta} i^{2} \mathbb{E}^{\mathcal{T}}\left[p_{i}^{u}(\mathcal{T})\right]}{\sum_{i=0}^{\Delta} i \mathbb{E}^{\mathcal{T}}\left[p_{i}^{u}(\mathcal{T})\right]} & =\frac{\mathbb{E}^{\mathcal{T}}\left[\phi(u)^{D^{*}-1}\right] \sum_{k=3}^{\Delta} p_{k} k(k-1) \phi(u)^{k-2}}{\sum_{k=3}^{\Delta} p_{k} k \phi(u)^{k-2}}+1 \\
& =m_{p}^{-1} \sum_{k=3}^{\Delta} p_{k} k(k-1) \phi(u)^{k-2}+1
\end{aligned}
$$

This completes the proof of the lemma.

\section{References}

[AB92] David J. Aldous and Mark Brown. Inequalities for rare events in timereversible Markov chains. I. In Stochastic inequalities (Seattle, WA, 1991), volume 22 of IMS Lecture Notes Monogr. Ser., pages 1-16. Inst. Math. Statist., Hayward, CA, 1992.

[ABG12] L. Addario-Berry, N. Broutin, and C. Goldschmidt. The continuum limit of critical random graphs. Probab. Theory Related Fields, 152(3-4):367-406, 2012.

[AF02] David Aldous and James Allen Fill. Reversible markov chains and random walks on graphs, 2002. Unfinished monograph, recompiled 2014, available at http://www. stat. berkeley.edu/ aldous/RWG/book.html.

[BLPS18] Nathanaël Berestycki, Eyal Lubetzky, Yuval Peres, and Allan Sly. Random walks on the random graph. Ann. Probab., 46(1):456-490, 2018.

[BS20] Shankar Bhamidi and Sanchayan Sen. Geometry of the vacant set left by random walk on random graphs, Wright's constants, and critical random graphs with prescribed degrees. Random Structures \& Algorithms, 56(3):676-721, 2020 .

[CF13] Colin Cooper and Alan Frieze. Component structure of the vacant set induced by a random walk on a random graph. Random Structures Algorithms, 42(2):135-158, 2013.

[ČT13] Jiři Černý and Augusto Teixeira. Critical window for the vacant set left by random walk on random regular graphs. Random Structures Algorithms, 43(3):313-337, 2013.

[ČTW11] Jiří Černý, Augusto Teixeira, and David Windisch. Giant vacant component left by a random walk in a random $d$-regular graph. Ann. Inst. Henri Poincaré Probab. Stat., 47(4):929-968, 2011. 
[Fri91] Joel Friedman. On the second eigenvalue and random walks in random $d$ regular graphs. Combinatorica, 11(4):331-362, 1991.

[FvdH17] Lorenzo Federico and Remco van der Hofstad. Critical window for connectivity in the configuration model. Combin. Probab. Comput., 26(5):660-680, 2017.

[Hae95] Willem H. Haemers. Interlacing eigenvalues and graphs. Linear Algebra Appl., 226/228:593-616, 1995.

[HM12] Hamed Hatami and Michael Molloy. The scaling window for a random graph with a given degree sequence. Random Structures Algorithms, 41(1):99-123, 2012 .

[JL09] Svante Janson and Malwina J. Luczak. A new approach to the giant component problem. Random Structures Algorithms, 34(2):197-216, 2009.

[LPW09] David A. Levin, Yuval Peres, and Elizabeth L. Wilmer. Markov chains and mixing times. American Mathematical Society, Providence, RI, 2009. With a chapter by James G. Propp and David B. Wilson.

[McD98] Colin McDiarmid. Concentration. In Probabilistic methods for algorithmic discrete mathematics, volume 16 of Algorithms Combin., pages 195-248. Springer, Berlin, 1998.

[MR95] Michael Molloy and Bruce Reed. A critical point for random graphs with a given degree sequence. In Proceedings of the Sixth International Seminar on Random Graphs and Probabilistic Methods in Combinatorics and Computer Science, "Random Graphs '93" (Poznań, 1993), volume 6, pages 161-179, 1995.

[SC97] Laurent Saloff-Coste. Lectures on finite Markov chains. In Lectures on probability theory and statistics (Saint-Flour, 1996), volume 1665 of Lecture Notes in Math., pages 301-413. Springer, Berlin, 1997.

[Tas10] Martin Tassy. Random interlacements on Galton-Watson trees. Electron. Commun. Probab., 15:562-571, 2010.

[Tei09] A. Teixeira. Interlacement percolation on transient weighted graphs. Electron. J. Probab., 14:no. 54, 1604-1628, 2009.

[vdH17a] Remco van der Hofstad. Random graphs and complex networks. Vol. 1. Cambridge Series in Statistical and Probabilistic Mathematics, [43]. Cambridge University Press, Cambridge, 2017.

[vdH17b] Remco van der Hofstad. Stochastic processes on random graphs. preliminary version of the book, available at https://www.win.tue.nl/ rhofstad/, 2017.

[Was15] Tobias Wassmer. Phase transition for the vacant set left by random walk on the giant component of a random graph. Ann. Inst. Henri Poincaré Probab. Stat., 51(2):756-780, 2015. 